\title{
Minimal Affinizations of Representations of Quantum Groups: the Rank 2 Case
}

By

\author{
Vyjayanthi CHARI ${ }^{*}$
}

\begin{abstract}
If $U_{q}(\mathfrak{g})$ is a finite-dimensional complex simple Lie algebra, an affinization of a finite-dimensional irreducible representation $V$ of $U_{q}(\mathfrak{g})$ is a finite-dimensional irreducible representation $\widehat{V}$ of $U_{q}(\hat{\mathfrak{g}})$ which contains $V$ with multiplicity one, and is such that all other $U_{q}(\mathrm{~g})$-types in $\widehat{V}$ have highest weights strictly smaller than that of $V$. We define a natural partial ordering $\checkmark$ on the set of affinizations of $V$. If $\mathrm{g}$ is of rank 2, we show that there is a unique minimal element with respect to this order and give its $U_{q}(\mathrm{~g})$-module structure when $\mathrm{g}$ is of type $A_{2}$ or $C_{2}$.
\end{abstract}

\section{Introduction}

If $g$ is a finite-dimensional complex simple Lie algebra, the associated 'untwisted' affine Lie algebra $\hat{\mathrm{g}}$ is a central extension, with one-dimensional centre, of the space of Laurent polynomial maps $\mathbf{C}^{\times} \rightarrow \mathrm{g}$ (on which a Lie bracket is defined using pointwise operations). Since the cocycle of the extension vanishes on the constant maps, we can regard $g$ as a subalgebra of $\hat{\mathrm{g}}$. If $V$ is any representation of $\mathfrak{g}$, it is easy to extend the action of $\mathfrak{g}$ on $V$ to an action of $\hat{\mathrm{g}}$ on the same space. If $a \in \mathbf{C}^{\times}$, evaluation at $a$ gives a homomorphism $e v_{a}: \hat{\mathrm{g}} \rightarrow \mathrm{g}$ (under which the centre maps to zero) which is the identity on $\mathrm{g}$, so pulling back $V$ by $e v_{a}$ gives the desired extension. It follows from the results of [2] that, if $V$ is finite-dimensional and irreducible, these are, up to isomorphism, the only possible extensions.

Quantum deformations $U_{q}(\mathfrak{g})$ and $U_{q}(\hat{\mathfrak{g}})$ of the universal enveloping algebras of $\mathfrak{g}$ and $\hat{g}$ were introduced in 1985 by V. G. Drinfel'd and M. Jimbo. These

Communicated by T. Miwa, January 18, 1995.

1991 Mathematics Subject Classification (s): 17B.

* Dept. of Math., Univ. of California, Riverside, CA92521, U.S.A.

Partially supported by the NSF. DMS-9207701. 
algebras depend on a parameter $q \in \mathbb{C}^{\times}$; we assume throughout this paper that $q$ is transcendental. It is well-known (see [5] or [10], for example) that, up to twisting by certain simple automorphisms, there is a natural one-to-one correspondence between the finite-dimensional representations of $U_{q}(\mathfrak{g})$ and those of g. Corresponding representations have the same character, and hence the same dimension. However, the structure of the finite-dimensional representations of $U_{q}(\hat{\mathfrak{g}})$ is not well-understood. A parametrization of these representations in the spirit of Cartan's highest weight classification of the finite-dimensional irreducible representations of $g$ is proved in the case $g=s l_{2}$ in [3], and in [6] in general.

As in the classical situation, we may regard $U_{q}(\mathrm{~g})$ as a subalgebra of $U_{q}(\hat{\mathfrak{g}})$. If $\mathrm{g}$ is of type $s l_{n}$, the action of $U_{q}(\mathfrak{g})$ on any representation $V$ extends to a representation of $U_{q}(\hat{\mathfrak{g}})$. However, if $\hat{\mathrm{g}}$ is not of type $s l_{n}$, it is not usually possible to extend the action of $U_{q}(\mathrm{~g})$ on an irreducible finite-dimensional represention $V$ to an action of $U_{q}(\hat{\mathrm{g}})$ on $V$. Thus, it is natural to ask how $V$ can be 'enlarged' so as to obtain a representation of $U_{q}(\hat{\mathrm{g}})$. To make this question precise, we define in this paper a natural partial ordering on the set of isomorphism classes of representations of $U_{q}(\mathfrak{g})$. By an affinization of a finitedimensional irreducible representation $V$ of $U_{q}(\mathfrak{g})$, we mean an irreducible representation $\widehat{V}$ of $U_{q}(\hat{\mathrm{g}})$ which contains $V$ as a $U_{q}(\mathfrak{g})$-subrepresentation with multiplicity one, and such that all other irreducible $U_{q}(\mathrm{~g})$-subrepresentations of $\widehat{V}$ are strictly smaller than $V$. (There is a clear analogy with the classical Harish Chandra theory of $(\mathfrak{g}, K)$-modules here.)

We prove that any given representation $V$ has only finitely many affinizations (at least one) up to $U_{q}(\mathrm{~g})$-isomorphism, and one may ask if any of them is 'canonical'. A reasonable interpretation of this question is to look for the minimal affinization (s) of $V$, with respect to our partial order. If $g=s l_{n}$, we show in [4] that every finite-dimensional irreducible representation of $U_{q}(\mathrm{~g})$ has, up to $U_{q}(\mathrm{~g})$-isomorphism, a unique minimal affinization. In this paper, we prove that, if $\mathrm{g}$ is of type $C_{2}$ or $G_{2}$, there is again a unique minimal affinization, and we describe it precisely in terms of the highest weight classification of representations of $U_{q}(\hat{\mathrm{g}})$ mentioned above. In contrast to the $s l_{n}$ case, the minimal affinization in these cases is not, in general, irreducible as a representation of $U_{q}(\mathrm{~g})$. In fact, in the $C_{2}$ case we describe the structure of all minimal affinizations as representations of $U_{q}(\mathrm{~g})$; a consequence of this result is that the minimal affinization of $V$ is irreducible under $U_{q}(\mathrm{~g})$ if and only if the value of its highest weight on the short simple root of $g$ is 0 or 1 . Subsequent papers will deal with the case when $g$ has rank greater that 2 .

The problem of constructing affinizations of representations of $U_{q}(\mathrm{~g})$ is important in several areas of mathematics and physics, as has been emphasized by I. B. Frenkel and N. Yu. Reshetikhin, among others (see Remark 4.2 in [9]). 
As one example, recall that, to any finite-dimensional irreducible representation $V$ of $U_{q}(\mathfrak{g})$ one can associate an R-matrix, i.e. an element $R \in \operatorname{End}(V \otimes V)$ which satisfies the 'quantum Yang-Baxter equation' (QYBE). There are many situations, however, in which it is important to have a solution of the 'QYBE with spectral parameters'. This is so, for example, in the theory of lattice models in statistical mechanics, for only when the R-matrix constructed from the Boltzmann weights of the model satisfies the QYBE with spectral parameters can one prove the existence of commuting transfer matrices and deduce the integrability of the model. (See [5], for example, for an introduction to these ideas.) Thus, it is natural to ask when $R$ can be 'embedded' in a parameter-dependent R-matrix $R(u) \in$ End $(V \otimes V)$. A sufficient condition for this is that the action of $U_{q}(\mathrm{~g})$ on $V$ extends to an action of $U_{q}(\hat{\mathrm{g}})$ on $V$, for then $V$ itself can be embedded in a 1-parameter family of representations of $U_{q}(\hat{\mathfrak{g}})$ by twisting with a certain 1-parameter family of automorphisms of $U_{q}(\hat{\mathfrak{g}})$ (which correspond, in the classical case, to 'rescaling' the $\mathbb{C}^{\times}$parameter in $\hat{\mathrm{g}}$ ).

A second example concerns the affine Toda field theory associated to $\hat{\mathrm{g}}$. This admits $U_{q}\left(\hat{\mathfrak{g}}^{*}\right)$ as a 'quantum symmetry group', where $\hat{\mathrm{g}}^{*}$ is the dual affine Lie algebra (whose generalized Cartan matrix is the transpose of that of $\hat{\mathrm{g}}$ ). It is well known that the classical solitons of this theory correspond essentially to the finite-dimensional irreducible representations of $\hat{\mathrm{g}}$. The solitons (or particle states) of the quantum theory should therefore correspond to the finitedimensional irreducible representations of $U_{q}\left(\hat{g}^{*}\right)$. Since not all representations of $U_{q}(\mathrm{~g})$ are affinizable on the same space, the quantum solitons come in 'multiplets', and there are generally 'more' quantum solitons than classical ones.

\section{§1. Quantum Affine Algebras}

Let $g$ be a finite-dimensional complex simple Lie algebra with Cartan subalgebra $\mathfrak{y}$ and Cartan matrix $A=\left(a_{i j}\right)_{i, j \in I}$. Fix coprime positive integers $\left(d_{i}\right)_{\imath \in I}$ such that $\left(d_{i} a_{i j}\right)$ is symmetric. Let $R$ be the set of roots and $R^{+}$a set of positive roots. The roots can be regarded as functions $I \rightarrow \mathbb{Z}$; in particular, the simple roots $\alpha_{i} \in R^{+}$are given by

$$
\alpha_{\imath}(j)=a_{j_{\imath}}, \quad(i, j \in I)
$$

Let $Q=\oplus_{i \in I} \mathbf{Z} . \alpha_{i} \subset \mathfrak{y}{ }^{*}$ be the root lattice, and set $Q^{+}=\sum_{i \in I} \mathbb{N} . \alpha_{i}$.

A weight is an arbitrary function $\lambda: I \rightarrow \mathbb{Z}$; denote the set of weights by $P$, and let

$$
P^{+}=\{\lambda \in P: \lambda(i) \geq 0 \text { for all } i \in I\}
$$


be the set of dominant weights. Define a partial order $\geq$ on $P$ by

$$
\lambda \geq \mu \quad \text { iff } \quad \lambda-\mu \in Q^{+} .
$$

Let $\theta$ be the unique highest root with respect to $\geq$.

Define a non-degenerate symmetric bilinear form $(, \quad)$ on $\mathfrak{y}^{*}$ by

$$
\left(\alpha_{i}, \alpha_{j}\right)=d_{i} a_{i j}
$$

and denote by $(, \quad)$ also the induced form on $\mathfrak{y}$. Set $d_{0}=\frac{1}{2}(\theta, \theta), a_{00}=2$, and, for all $i \in I$,

$$
a_{0 i}=-\frac{2\left(\theta, \alpha_{i}\right)}{(\theta, \theta)}, \quad a_{i 0}=-\frac{2\left(\theta, \alpha_{i}\right)}{\left(\alpha_{i}, \alpha_{i}\right)}
$$

Let $\widehat{I}=I \amalg\{0\}$ and $\widehat{A}=\left(a_{i j}\right)_{i, j \in I}$. Then, $\widehat{A}$ is the generalized Cartan matrix of the untwisted affine Lie algebra $\hat{g}$ associated to $g$.

From Section 5 onwards, we shall be interested in the case when $\mathfrak{g}$ is of type $C_{2}$. Then,

$$
\begin{gathered}
I=\{1,2\}, \quad d_{0}=d_{1}=2, \quad d_{2}=1, \quad \theta=\alpha_{1}+2 \alpha_{2}, \\
A=\left(\begin{array}{cc}
2 & -1 \\
-2 & 2
\end{array}\right), \quad \widehat{A}=\left(\begin{array}{ccc}
2 & 0 & -1 \\
0 & 2 & -1 \\
-1 & -2 & 2
\end{array}\right),
\end{gathered}
$$

the rows of $\widehat{A}$ being numbered $0,1,2$.

Let $q \in \mathbf{C}^{\times}$be transcendental, and, for $r, n \in \mathbb{N}, n \geq r$, define

$$
\begin{aligned}
& {[n]_{q}=\frac{q^{n}-q^{-n}}{q-q^{-1}},} \\
& {[n]_{q} !=[n]_{q}[n-1]_{q \ldots}[2]_{q}[1]_{q},} \\
& {\left[\begin{array}{l}
n \\
r
\end{array}\right]_{q}=\frac{[n]_{q} !}{[r]_{q} ![n-r]_{q} !} .}
\end{aligned}
$$

If $i \in \widehat{I}$, let $q_{i}=q^{d_{i}}$.

Definition 1.1. With the above notation, $U_{q}(\hat{\mathfrak{g}})$ is the unital associative algebra over $\mathbf{C}$ with generators $x_{i}^{ \pm}, k_{i}^{ \pm 1}(i \in \widehat{I})$, and the following defining relations: 


$$
\begin{gathered}
k_{i} k_{i}^{-1}=k_{i}^{-1} k_{i}=1, \quad k_{i} k_{j}=k_{j} k_{i}, \\
k_{i} x_{j}^{ \pm} k_{i}^{-1}=q_{i}^{ \pm a_{i}} x_{j}^{ \pm}, \\
{\left[x_{i}^{+}, x_{j}^{-}\right]=\delta_{i j} \frac{k_{i}-k_{i}^{-1}}{q_{i}-q_{i}^{-1}},} \\
\sum_{r=0}^{1-a_{i} y}\left[\begin{array}{c}
1-a_{i j} \\
r
\end{array}\right]_{q_{i}}\left(x_{\imath}^{ \pm}\right)^{r} x_{j}^{ \pm}\left(x_{i}^{ \pm}\right)^{1-a_{i j}-r}=0, \quad i \neq j .
\end{gathered}
$$

The algebra with generators $x_{i}^{ \pm}, k_{i}^{ \pm 1}(i \in I)$ and the above defining relations (with the indices $i, j$ restricted to $I$ ) is denoted by $U_{q}(\mathfrak{g})$.

Note that there is a canonical homomorphism of algebras $U_{q}(\mathfrak{g}) \rightarrow U_{q}(\hat{\mathfrak{g}})$ which takes $x_{i}^{ \pm} \rightarrow x_{i}^{ \pm}, k_{i}^{ \pm} \rightarrow k_{i}^{ \pm}$for all $i \in I$. The following result is well-known (see [5], for example).

Proposition 1.2. $U_{q}(\hat{\mathfrak{g}})$ has the structure of a Hopf algebra, with comultiplication $\Delta$, counit $\epsilon$, and antipode $S$, given by

$$
\begin{gathered}
\Delta\left(x_{i}^{+}\right)=x_{i}^{+} \otimes k_{i}+1 \otimes x_{i}^{+}, \\
\Delta\left(x_{i}^{-}\right)=x_{\imath}^{-} \otimes 1+k_{i}^{-1} \otimes x_{\imath}^{-}, \\
\Delta\left(k_{\imath}^{ \pm 1}\right)=k_{i}^{ \pm 1} \otimes k_{i}^{ \pm 1}, \\
\epsilon\left(x_{i}^{ \pm}\right)=0, \quad \epsilon\left(k_{\imath}^{ \pm 1}\right)=1, \\
S\left(x_{\imath}^{+}\right)=-x_{\imath}^{+} k_{\imath}^{-1}, \quad S\left(x_{i}^{-}\right)=-k_{\imath} x_{\imath}^{-}, \quad S\left(k_{\imath}^{ \pm 1}\right)=k_{\imath}^{\mp 1},
\end{gathered}
$$

for all $i \in \widehat{I}$. Moreover, $U_{q}(\mathrm{~g})$ is a Hopf algebra with structure maps given by the same formulas, but with the index $i$ being restricted to the set $I$.

It is well-known that $\hat{\mathrm{g}}$ may also be described as a central extension, with one-dimensional centre, of the loop algebra of $\mathfrak{g}$, i.e. the space of Laurent polynomial maps $\mathbf{C}^{\times} \rightarrow \mathrm{g}$ under pointwise operations. Drinfel'd [7] and Beck [1] give an analogous realization of $U_{q}(\hat{\mathrm{g}})$ :

Theorem 1.3. Let $\mathscr{A}_{q}$ be the unital associative algebra with generators $x_{l, r}^{ \pm}$ (i $\in I, r \in \mathbf{Z}), k_{\imath}^{ \pm 1}(i \in I), h_{\imath, r}(i \in I, r \in \mathbf{Z} \backslash\{0\})$ and $c^{ \pm 1 / 2}$, and the following defining relations:

$$
\begin{gathered}
c^{ \pm 1 / 2} \text { are central }, \\
k_{\imath} k_{\imath}^{-1}=k_{\imath}^{-1} k_{\imath}=1, c^{1 / 2} c^{-1 / 2}={ }^{-1 / 2} c^{1 / 2}=1, \\
k_{\imath} k_{j}=k_{j} k_{\imath}, k_{i} h_{j, r}=h_{j, r} k_{\imath},
\end{gathered}
$$




$$
\begin{gathered}
k_{i} x_{j, r}^{ \pm} k_{i}^{-1}=q_{i}^{ \pm a_{i v}} x_{j, r}^{ \pm}, \\
{\left[h_{i, r}, h_{j, s}\right]=\delta_{r,-s} \frac{1}{r}\left[r a_{i j}\right]_{q_{i}} \frac{c^{r}-c^{-r}}{q_{j}-q_{j}^{-1}},} \\
{\left[h_{i, r}, x_{j, s}^{ \pm}\right]= \pm \frac{1}{r}\left[r a_{i j}\right]_{q_{t}} c^{\mp|r| / 2} x_{j, r+s}^{ \pm},} \\
x_{i, r+1}^{ \pm} x_{j, s}^{ \pm}-q_{i}^{ \pm a_{i \jmath}} x_{j, s}^{ \pm} x_{i, r+1}^{ \pm}=q_{i}^{ \pm a_{i j}} x_{i, r}^{ \pm} x_{j, s+1}^{ \pm}-x_{j, s+1}^{ \pm} x_{i, r}^{ \pm}, \\
{\left[x_{i, r}^{+}, x_{j, s}^{-}\right]=\delta_{i j} \frac{c^{(r-s) / 2} \phi_{i, r+s}^{+}-c^{-(r-s) / 2} \phi_{i, r+s}^{-}}{q_{i}-q_{i}^{-1}},} \\
\sum_{\pi \in \Sigma m} \sum_{k=0}^{m}(-1)^{k}\left[\begin{array}{c}
m \\
k
\end{array}\right]_{q_{i}} x_{i, r \pi(1)}^{ \pm} x_{i, r \pi(k)}^{ \pm} x_{j, s}^{ \pm} x_{i, r \pi(k+1)}^{ \pm} \ldots x_{i, r \pi(m)}^{ \pm}=0, i \neq j,
\end{gathered}
$$

for all sequences of integers $r_{1}, \ldots, r_{m}$, where $m=1-a_{i j}, \sum_{m}$ is the symmetric group on $m$ letters, and the $\phi_{i, r}^{ \pm}$are determined by equating powers of $u$ in the formal power series

$$
\sum_{r=0}^{\infty} \phi_{i, \pm r}^{ \pm} u^{ \pm r}=k_{i}^{ \pm 1} \exp \left( \pm\left(q_{i}-q_{i}^{-1}\right) \sum_{s=1}^{\infty} h_{i, \pm s} u^{ \pm s}\right)
$$

If $\theta=\sum_{i \in I} m_{i} \alpha_{i}$, set $k_{\theta}=\Pi_{i \in I} k_{i}^{m_{z}}$. Suppose that the root vector $\bar{x}_{\theta}^{+}$of $\mathrm{g}$ corresponding to $\theta$ is expressed in terms of the simple root vectors $\bar{x}_{i}^{+}(i \in I)$ of $\mathfrak{g}$ as

$$
\bar{x}_{\theta}^{+}=\lambda\left[\bar{x}_{i_{1}}^{+},\left[\bar{x}_{i_{2}}^{+}, \ldots,\left[\bar{x}_{i_{k}}^{+}, \bar{x}_{j}^{+}\right] \ldots\right]\right]
$$

for some $\lambda \in \mathbb{C}^{\times}$. Define maps $w_{i}^{ \pm}: U_{q}(\hat{\mathfrak{g}}) \rightarrow U_{q}(\hat{\mathfrak{g}})$ by

$$
w_{i}^{ \pm}(a)=x_{i, 0}^{ \pm} a-k_{i}^{ \pm 1} a k_{i}^{\mp 1} x_{i, 0}^{ \pm} .
$$

Then, there is an isomorphism of algebras $f: U_{q}(\hat{\mathrm{g}}) \rightarrow \mathscr{A}_{q}$ defined on generators by

$$
\begin{aligned}
f\left(k_{0}\right)=k_{\theta}^{-1}, f\left(k_{i}\right) & =k_{i}, f\left(x_{i}^{ \pm}\right)=x_{i, 0}^{ \pm}, \quad(i \in I), \\
f\left(x_{0}^{+}\right) & =\mu w_{i_{1}}^{-} \ldots w_{i_{k}}^{-}\left(x_{\bar{j}, 1}^{-}\right) k_{\theta}^{-1}, \\
f\left(x_{0}^{-}\right) & =\lambda k_{\theta} w_{i_{1}}^{+} \ldots w_{i_{k}}^{+}\left(x_{j,-1}^{+}\right),
\end{aligned}
$$

where $\mu \in \mathbb{C}^{\times}$is determined by the condition

$$
\left[x_{0}^{+}, x_{0}^{-}\right]=\frac{k_{0}-k_{0}^{-1}}{q_{0}-q_{0}^{-1}} .
$$


Let $\widehat{U}^{ \pm}$(resp. $\widehat{U}^{0}$ ) be the subalgebra of $U_{q}(\hat{\mathfrak{g}})$ generated by the $x_{i, r}^{ \pm}$(resp. by the $\phi_{i, r}^{ \pm}$) for all $i \in I, r \in \mathbb{Z}$. Similarly, let $U^{ \pm}$(resp. $U^{0}$ ) be the subalgebra of $U_{q}(\mathrm{~g})$ generated by the $x_{i}^{ \pm}$(resp. by the $k_{\imath}^{ \pm 1}$ ) for all $i \in I$. It is not difficult to prove

Proposition 1.4. (a) $U_{q}(\mathrm{~g})=U^{-} \cdot U^{0} \cdot U^{+}$.

(b) $U_{q}(\hat{\mathfrak{g}})=\widehat{U}^{-} \cdot \widehat{U}^{0} . \widehat{U}^{+}$.

It is clear that setting

$$
\operatorname{deg}\left(x_{i, r}^{ \pm}\right)=\operatorname{deg}\left(h_{i, r}\right)=r, \quad \operatorname{deg}\left(c^{ \pm 1 / 2}\right)=\operatorname{deg}\left(k_{i}^{ \pm 1}\right)=0, \quad(i \in I, r \in \mathbf{Z})
$$

gives $U_{q}(\hat{\mathfrak{g}})$ the structure of a graded algebra. The following result is a more precise formulation of this remark.

Proposition 1.5. For all $t \in \mathbf{C}^{\times}$, there exists a Hopf algebra automorphism $\tau_{t}$ of $U_{q}(\hat{\mathfrak{g}})$ such that

$$
\begin{aligned}
& \tau_{t}\left(x_{i, r}^{ \pm}\right)=t^{r}\left(x_{i, r}^{ \pm}\right), \quad \tau_{t}\left(h_{i, r}\right)=t^{r} h_{i, r}, \\
& \tau_{t}\left(k_{t}^{ \pm 1}\right)=k_{\imath}^{ \pm 1}, \quad \tau_{t}\left(c^{ \pm 1 / 2}\right)=c^{ \pm 1 / 2} .
\end{aligned}
$$

Proof. It is clear, as we have already said, that there is an algebra automorphism $\tau_{t}$ given on generators by the above formulas. To see that $\tau_{t}$ respects the coalgebra structure, note that, by the formula for the isomorphism $f$ in 1.3 ,

$$
\begin{aligned}
& \tau_{t}\left(x_{i}^{ \pm}\right)=x_{i}^{ \pm}, \quad \tau_{t}\left(k_{i}^{ \pm 1}\right)=k_{\imath}^{ \pm 1}, \quad(i \in I) \\
& \tau_{t}\left(x_{0}^{ \pm}\right)=t^{\mp 1} x_{0}^{ \pm}, \quad \tau_{t}\left(k_{0}^{ \pm 1}\right)=k_{0}^{ \pm 1} .
\end{aligned}
$$

Using 1.2 , it is easy to check that both sides of the equations

$$
\left(\tau_{t} \otimes \tau_{t}\right) \circ \Delta=\Delta \circ \tau_{t}, \quad \tau_{t} \circ S=S \circ \tau_{t}
$$

agree on the generators in 1.1 , and hence on the whole of $U_{q}(\hat{\mathrm{g}})$.

The $\tau_{t}$ are the quantum analogues of the 'translation' automorphisms which take a loop $\boldsymbol{\ell}: \mathbf{C}^{\times} \rightarrow \mathrm{g}$ to the loop $\boldsymbol{\ell}_{t}$ given by $\boldsymbol{\ell}_{t}(u)=\boldsymbol{\ell}(t u)$.

We shall also need to make use of the quantum analogue of the Cartan in volution of $\hat{\mathrm{g}}$ : 
Proposition 1.6. There is a unique algebra involution $\widehat{\omega}$ of $U_{q}(\hat{\mathfrak{g}})$ given on the generators of the presentation 1.3 by

$$
\begin{gathered}
\widehat{\omega}\left(x_{i, r}^{ \pm}\right)=-x_{i,-r}^{\mp}, \quad \widehat{\omega}\left(h_{i, r}\right)=-h_{i,-r}, \\
\widehat{\omega}\left(\phi_{i, r}^{ \pm}\right)=\phi_{i,-r}^{\mp}, \quad \widehat{\omega}\left(k_{i}^{ \pm 1}\right)=k_{i}^{\mp 1}, \\
\widehat{\omega}\left(c^{ \pm 1 / 2}\right)=c^{\mp 1 / 2} .
\end{gathered}
$$

Moreover, we have

$$
\begin{gathered}
(\widehat{\omega} \otimes \widehat{\omega}) \circ \Delta=\Delta^{o p} \circ \widehat{\omega}, \\
\widehat{\omega}^{-1} \circ S^{-1} \circ \widehat{w} \circ S=\kappa,
\end{gathered}
$$

where $\Delta^{o p}$ is the opposite comultiplication of $U_{q}(\hat{\mathfrak{g}})$ and $\kappa$ is the Hopf algebra automorphism of $U_{q}(\hat{\mathfrak{g}})$ such that

$$
\kappa\left(x_{i}^{ \pm}\right)=q_{\imath}^{ \pm 2} x_{i}^{ \pm}, \quad \kappa\left(k_{i}^{ \pm 1}\right)=k_{\imath}^{ \pm 1} \quad(i \in I) .
$$

Proof. That the formulas in (2) do define an algebra involution of $U_{q}(\hat{\mathrm{g}})$ is easily checked, using 1.3. To prove (3) and (4), we compute $\widehat{\omega}\left(x_{0}^{ \pm}\right)$. Note that, for any $a \in U_{q}(\hat{\mathfrak{g}})$,

$$
\begin{aligned}
\widehat{\omega}\left(w_{i}^{ \pm}(a)\right) & =\widehat{\omega}\left(x_{i, 0}^{ \pm} a-k_{i}^{ \pm 1} a k_{i}^{\mp 1} x_{i, 0}^{ \pm}\right) \\
& =-\left(x_{i, 0}^{\mp} \widehat{\omega}(a)-k_{i}^{\mp 1} \widehat{\omega}(a) k_{i}^{\mp 1} x_{i, 0}^{\mp}\right) \\
& =-w_{i}^{\mp}(\widehat{\omega}(a)) .
\end{aligned}
$$

It follows from the formula for the isomorphism $f$ in 1.3 that

$$
\begin{aligned}
\widehat{\omega}\left(x_{0}^{+}\right) & =-\mu(-1)^{k} w_{i_{1} \ldots}^{+} w_{i_{k}}^{+}\left(x_{j,-1}^{+}\right) k_{\theta} \\
& =-\lambda^{-1} \mu(-1)^{k} q_{0}^{2} x_{0}^{-},
\end{aligned}
$$

and, because $\widehat{\omega}$ is an involution,

$$
\widehat{\omega}\left(x_{0}^{-}\right)=-\lambda \mu^{-1}(-1)^{k} q_{0}^{-2} x_{0}^{+}
$$

Equations (3) and (4) are now easily checked on the generators in 1.1.

It is clear that $\widehat{\omega}$ is compatible, via the canonical map $U_{q}(\mathfrak{g}) \rightarrow U_{q}(\hat{\mathfrak{g}})$, with the Cartan involution $\omega$ of $U_{q}(\mathfrak{g})$, given by

$$
\omega\left(x_{i}^{ \pm}\right)=-x_{i}^{\mp}, \quad \omega\left(k_{i}^{ \pm 1}\right)=k_{i}^{\mp 1} \quad(i \in I) .
$$




\section{§2. Finite-dimensional Representations}

Let $W$ be a representation of $U_{q}(\mathrm{~g})$, i.e. a (left) $U_{q}(\mathrm{~g})$-module. One says that $\lambda \in P$ is a weight of $W$ if the weight space

$$
W_{\lambda}=\left\{w \in W \mid k_{i} . w=q_{i}^{\lambda(t)} w\right\}
$$

is non-zero; the set of weights of $W$ is denoted by $P(W)$. We say that $W$ is of type 1 if

$$
W=\bigoplus_{\lambda \in P(W)} W_{\lambda}
$$

The character of $W$ is the function $c h_{W} P \longrightarrow \mathbf{N}$ given by $c h_{W}(\lambda)=\operatorname{dim}\left(W_{\lambda}\right)$.

If $W$ is representation of $U_{q}(\mathrm{~g})$, one says that $w \in W_{\lambda}$ is a highest weight vector if $x_{\imath}^{+} . w=0$ for all $i \in I$. If $W=U_{q}(\mathfrak{g}) . w$, one says that $W$ is a highest weight representation with highest weight $\lambda$. Lowest weight vectors and representations are defined similarly, by replacing $x_{\imath}^{+}$by $x_{\imath}^{-}$.

For a proof of the following proposition, see [5] or [10].

Proposition 2.1. (a) Every finite-dimensional representation of $U_{q}(\mathrm{~g})$ is completely reducible.

(b) Every finite-dimensional irreducible representation of $U_{q}(\mathrm{~g})$ can be obtained from a type 1 representation by twisting with an automorphism of $U_{q}(\mathrm{~g})$.

(c) Every finite-dimensional irreducible representation of $U_{q}(\mathrm{~g})$ of type 1 is both highest and lowest weight. Assigning to such a representation its highest weight defines a bijection between the set of isomorphism classes of finite-dimensional irreducible representations of $U_{q}(\mathfrak{g})$ and $P^{+}$.

(d) The finite-dimensional irreducible representation $V(\lambda)$ of $U_{q}(\mathrm{~g})$ of highest weight $\lambda \in P^{+}$has the same character as the irreducible representation of $\mathrm{g}$ of the same highest weight.

By (a) and (c), if $W$ is any finite-dimensional representation of $U_{q}(\mathrm{~g})$ of type 1 , we can write

$$
W \cong \bigoplus_{\lambda \in P^{+}} V(\lambda) \oplus m_{\lambda}(W)
$$

for some uniquely determined multiplicities $m_{\lambda}(W) \in \mathbf{N}$. It will be useful to define $m_{\lambda}(W)=0$ for $\lambda \in P \backslash P^{+}$. 
Proposition 2.1 (continued). (e) The multiplicities of the irreducible components in the tensor product $V(\lambda) \otimes V(\mu)$, where $\lambda, \mu \in P^{+}$, is the same as in the tensor product of the irreducible representation of $\mathrm{g}$ of the same highest weight.

We now turn to representations of $U_{q}(\hat{\mathfrak{g}})$. Note that such a representation $V$ may be regarded as a representation of $U_{q}(\mathfrak{g})$ via the canonical homomorphism $U_{q}(\mathfrak{g}) \rightarrow U_{q}(\hat{\mathrm{g}})$. We say that $V$ is of type 1 if $c^{1 / 2}$ acts as the identity on $V$, and if $k_{i}$ acts semisimply on $V$ for all $i \in \widehat{I}$. Observe that $V$ is then of type 1 as a representation of $U_{q}(\mathfrak{g})$; in particular, the multiplicities $m_{\lambda}(V)(\lambda \in P)$ are well-defined.

A vector $v \in V$ is a highest weight vector if

$$
x_{i, r .}^{+} v=0, \quad \phi_{i, r .}^{ \pm} v=\Phi_{i, r}^{ \pm} v, \quad c^{1 / 2} . v=v,
$$

for some complex numbers $\Phi_{i, r}^{ \pm}$. If, in addition, $V=U_{q}(\hat{\mathfrak{g}}) . v$, then $V$ is called a highest weight representation, and the pair of $(I \times \mathbb{Z})$-tuples $\left(\Phi_{i, r}^{ \pm}\right)_{\imath \in I, r \in \mathbf{Z}}$ its highest weight. Note that $\Phi_{i, r}^{+}=0$ (resp. $\left.\Phi_{i, r}^{-}=0\right)$ if $r<0$ (resp. if $r>0$ ), and that $\Phi_{i, 0}^{+} \Phi_{i, 0}^{-}=1$. (In [5], highest weight representations of $U_{q}(\hat{\mathfrak{g}})$ are called 'pseudo-highest weight'.) Lowest weight vectors and representations of $U_{q}(\hat{\mathfrak{g}})$ are defined similarly.

The following result is proved in [5].

Proposition 2.2. (a) Every finite-dimensional irreducible representation of $U_{q}(\hat{\mathfrak{g}})$ can be obtained from a type 1 representation by twisting with an automorphism of $U_{q}(\hat{\mathfrak{g}})$.

(b) Every finite-dimensional irreducible representation of $U_{q}(\hat{\mathrm{g}})$ of type 1 is both highest and lowest weight.

Note, however, that in contrast to the case of $U_{q}(\mathfrak{g})$, finite-dimensional representations of $U_{q}(\hat{\mathfrak{g}})$ are not completely reducible, in general.

The next result gives a parametrization of the finite-dimensional irreducible representations of $U_{q}(\hat{\mathfrak{g}})$ of type 1 analogous to that given for $U_{q}(\mathfrak{g})$ by 2.1 (c). If $\mathbb{P}=\left(P_{i}\right)_{i \in I}$ is any $I$-tuple of polynomials $P_{i} \in \mathbb{C}[u]$, its degree $\operatorname{deg}(\mathbb{P}) \in$ $P^{+}$is defined by

$$
\operatorname{deg}(\mathbb{P})(i)=\operatorname{deg}\left(P_{i}\right)
$$

Let $\mathscr{P}$ be the set of $I$-tuples of polynomials with constant term 1 , and, for any $\lambda$ $\in P^{+}$, let

$$
\mathscr{P}^{\lambda}=\{\mathbb{P} \in \mathscr{P} \mid \operatorname{deg}(\mathbb{P})=\lambda\}
$$


Theorem 2.3. Let $V$ be a finite-dimensional irreducible representation of $U_{q}(\hat{\mathfrak{g}})$ of type 1 and highest weight $\left(\Phi_{i, r}^{ \pm}\right)_{i \in I, r \in \mathbf{Z} .}$ Then, there exists $\mathbf{P}=\left(P_{i}\right)_{i \in I} \in$ $\mathscr{P}$ such that

$$
\sum_{r=0}^{\infty} \Phi_{i, r}^{+} u^{r}=q_{i}^{d e g}\left(P_{s}\right) \frac{P_{i}\left(q_{i}^{-2} u\right)}{P_{i}(u)}=\sum_{r=0}^{\infty} \Phi_{i, r}^{-} u^{-r}
$$

in the sense that the left-and right-hand terms are the Laurent expansions of the middle term about 0 and $\infty$, respectively. Assigning to $V$ the I-tuple $\mathbf{P}$ defines a bijection between the set of isomorphism classes of finite-dimensional irreducible representation of $U_{q}(\hat{\mathfrak{g}})$ of type 1 and $\mathscr{P}$.

This result is proved in [3] when $g=s l_{2}(\mathbf{C})$, in [5] when $g=s l_{n}(\mathbf{C})$, and in [6] in the general case. We denote by $V(\mathbf{P})$ the finite-dimensional irreducible representation of $U_{q}(\hat{\mathfrak{g}})$ associated to $\mathbf{P} \in \mathscr{P}$. Abusing notation, we shall say that a representation $V$ as in 2.3 has highest weight $\mathbf{P}$.

The next result describes the behaviour of the representations $V(\mathbf{P})$ under tensor products. If $\mathbf{P}=\left(P_{i}\right)_{i \in I}, \mathbf{Q}=\left(Q_{i}\right)_{i \in I} \in \mathscr{P}$, let $\mathbf{P} \otimes \mathbf{Q} \in \mathscr{P}$ be the $I$-tuple $\left(P_{i} Q_{i}\right)_{i \in I}$. Obviously, $\operatorname{deg}(\mathbf{P} \otimes \mathbf{Q})=\operatorname{deg}(\mathbf{P})+\operatorname{deg}(\mathbf{Q})$.

Proposition 2.4. Let $\mathbf{P}, \mathbf{Q} \in \mathscr{P}$ be as above, and let $v_{\mathbf{P}}$ and $v_{\mathbf{Q}}$ be highest weight vectors of $V(\mathbf{P})$ and $V(\mathbf{Q})$, respectively. Then, in $V(\mathbf{P}) \otimes V(\mathbf{Q})$,

$$
x_{i, r}^{+}\left(v_{\mathbf{P}} \otimes v_{\mathbf{Q}}\right)=0, \quad \phi_{i, r}^{ \pm}\left(v_{\mathbf{P}} \otimes v_{\mathbf{Q}}\right)=\Psi_{i, r}^{ \pm}\left(v_{\mathbf{P}} \otimes v_{\mathbf{Q}}\right),
$$

where the complex numbers $\Psi_{\imath, r}^{ \pm}$are related to the polynomials $P_{i} Q_{\imath}$ as the $\Phi_{i, r}^{ \pm}$are related to $P_{\imath}$ in (5).

The proof is essentially the same as that given in [3] when $g=s l_{2}(\mathbf{C})$.

Corollary 2.5. If $\mathbf{P}, \mathbf{Q} \in \mathscr{P}, V(\mathbf{P} \otimes \mathbf{Q})$ is isomorphic to a quotient of the subrepresentation of $V(\mathbf{P}) \otimes V(\mathbf{Q})$ generated by the tensor product of the highest weight vectors.

Let $\lambda_{i}(i \in I)$ be the fundamental weights of $\mathrm{g}$ :

$$
\lambda_{i}(j)=\delta_{i j} \quad(i, j \in I) .
$$

For any $a \in \mathbf{C}^{\times}$, let $V\left(\lambda_{i}, a\right)=V(\mathbf{P})$, where

$$
P_{j}(u)= \begin{cases}1-a^{-1} u & \text { if } j=i \\ 1 & \text { if } j \neq i\end{cases}
$$


The $V\left(\lambda_{i}, a\right)$ are called the fundamental representations of $U_{q}(\hat{\mathfrak{g}})$. This terminology is justified by the following consequence of 2.5 :

Corollary 2.6. Every finite-dimensional irreducible representation of $U_{q}(\hat{\mathfrak{g}})$ of type 1 is isomorphic to a subquotient of a tensor product of fundamental representations.

\section{§3. Minimal Affinizations}

We propose the following definition.

Definition 3.1. If $\lambda \in P^{+}$, a finite-dimensional irreducible representation $V$ of $U_{q}(\hat{\mathfrak{g}})$ of type 1 is said to be an affinization of $\lambda$ if the highest weight $\mathbb{P}$ of $V$ satisfies deg $(\mathbb{P})=\lambda$. Two affinizations $V$ and $V^{\prime}$ of $\lambda$ are said to be equivalent if $V$ and $V^{\prime}$ are isomorphic as representation of $U_{q}(\mathrm{~g})$.

Remark 3.2. It follows from 1.3 that, if $V$ is an affinization of $\lambda$, then

$$
V \cong V(\lambda) \oplus \bigoplus_{\left\{\mu \in P^{+} \mid \mu<\lambda\right\}} V(\mu)^{m_{\mu}(V)}
$$

as a representation of $U_{q}(\mathrm{~g})$. Thus, $V$ gives a way of 'extending' the action of $U_{q}(\mathrm{~g})$ on $V(\lambda)$ to an action of $U_{q}(\hat{\mathrm{g}})$, at the expense of 'enlarging' $V(\lambda)$ by adding representations of $U_{q}(\mathrm{~g})$ of smaller highest weight.

If $V$ is an affinization of $\lambda$, we denote its equivalence class by $[V]$, and we write $\mathscr{Q}^{\lambda}$ for the set of equivalence classes of affinizations of $\lambda$. Note that there is an obvious surjective map $\mathscr{P}^{\lambda} \rightarrow \mathscr{Q}^{\lambda}$, given by $\mathbf{P} \mapsto[V(\mathbb{P})]$.

One can easily describe $\mathscr{Q}^{\lambda}$ in case $\lambda$ is fundamental:

Proposition 3.3. For any $i \in I$,

$$
\mathscr{Q}^{\lambda_{i}}=\left\{\left[V\left(\lambda_{i}, 1\right)\right]\right\} .
$$

Proof. We need the following lemma, which will also be used elsewhere:

Lemma 3.4. Let $\rho: U_{q}(\hat{\mathfrak{g}}) \rightarrow$ End $(V)$ be a finite-dimensional irreducible representation of type 1 with highest weight $\mathbf{P}=\left(P_{i}\right)_{i \in I}$. For any $t \in \mathbb{C}^{\times}$, denote by $\tau_{t}^{*}(V)$ the representation $\rho \circ \tau_{t}$. Then, $\tau_{t}^{*}(V)$ has highest weight $\mathbb{P}^{t}=\left(P_{i}^{t}\right)_{i \in I}$, where 


$$
P_{i}^{t}(u)=P_{i}(t u)
$$

Proof. This is immediate from (5), since

$$
\tau_{t}\left(\phi_{i, r}^{ \pm}\right)=t^{ \pm r} \phi_{i, r}^{ \pm} \quad(i \in I, r \in \mathbf{Z})
$$

If $V$ is an affinization of $\lambda_{j}$, with highest weight $\mathbf{P}=\left(P_{i}\right)_{i \in I}$, say, then $\operatorname{deg}\left(P_{i}\right)=\lambda_{j}(i)=\delta_{i j}$, so $V \cong V\left(\lambda_{j}, t\right)$ as representations of $U_{q}(\hat{\mathfrak{g}})$, for some $t \in \mathbf{C}^{\times}$. But then $\tau_{t}^{*}(V) \cong V\left(\lambda_{j}, 1\right)$. In particular, $V \cong V\left(\lambda_{j}, 1\right)$ as representations of $U_{q}(\mathrm{~g})$. This proves 3.3 .

For arbitrary $\lambda \in P^{+}$, we have

Proposition 3.5. For any $\lambda \in P^{+}, \mathscr{Q}^{\lambda}$ is a finite set.

Proof. Let $V$ be an affinization of $\lambda$, let $\mathbf{P} \in \mathscr{P}^{\lambda}$ be the highest weight of $V$, and suppose that

$$
P_{\imath}(u)=\prod_{r=1}^{\lambda(\imath)}\left(1-a_{\imath, r}^{-1} u\right)
$$

where $a_{\imath, r} \in \mathbf{C}^{\times}$. By 2.5, $V$ is isomorphic to a subquotient of

$$
\bigotimes_{\imath \in I}\left(\bigotimes_{r=1}^{\lambda(\imath)} V\left(\lambda_{i}, a_{i, r}\right)\right)
$$

(the terms in the tensor products may be taken in any order). By 2.1 (a) and $3.4, V$ is isomorphic as a representation of $U_{q}(\mathfrak{g})$ to a subrepresentation of

$$
\bigotimes_{i \in I} V\left(\lambda_{i}, 1\right)^{\otimes \lambda(i)}
$$

Up to isomorphism, this representation obviously has only finitely many subrepresentations, hence 3.5 is proved.

The rest of this section is devoted to the definition of a natural partial order on $\mathscr{Q}^{\lambda}$. It is convenient to first define a partial order on a set which contains all of the $\mathscr{Q}^{\lambda}$. Namely, if $f: P^{+} \rightarrow \mathbf{N}$ is any function, let

$$
\operatorname{supp}(f)=\left\{\lambda \in P^{+} \mid f(\lambda)>0\right\}
$$


and define

$$
\mathscr{F}=\left\{f \in \mathbb{N}^{P^{+}} \mid \operatorname{supp}(f) \text { is finite }\right\}
$$

Definition 3.6. Let $f, g \in \mathscr{F} . \quad$ We say that $f \preceq g$ iff, for all $\mu \in P^{+}$, either

(i) $f(u) \leq g(\mu)$, or

(ii) there exists $\nu>\mu$ with $f(\nu)<g(\nu)$.

\section{Proposition 3.7. Sis a partial order on $\mathscr{F}$.}

Proof. That $f \preceq f$, for all $f \in \mathscr{F}$, is obvious. If $f \preceq g$ and $g \preceq f$, suppose for a contradiction that there exists $\mu \in P^{+}$with $f(\mu) \neq g(\mu)$. Note that, since $\operatorname{supp}(f) \cup \operatorname{supp}(g)$ is finite, there are at most finitely many such $\mu$, so we may assume that $\mu$ is maximal (with respect to the partial order on $P^{+}$) among those weights for which $f(\mu) \neq g(\mu)$. Without loss, assume that $f(\mu)<g(\mu)$. Since $g \leq f$, there exist $\nu>\mu$ with $f(\nu)>g(\nu)$; but this contradicts the maximality of $\mu$.

Suppose finally that $f, g, h \in \mathscr{F}$ are such that $f \preceq g$ and $g \preceq h$, and assume for a contradiction that $f \Varangle h$. This means that there exists $\mu \in P^{+}$such that

$$
f(\mu)>h(\mu) \text { and, for all } \nu>\mu, f(\nu) \geq h(\nu) .
$$

If 3.6 (i) holds for $f$ and $g$, then by (6), $g(\mu)>h(\mu)$. Since $g \leftrightharpoons h$, there exists $\nu^{\prime}>\mu$ with $g\left(\nu^{\prime}\right)<h\left(\nu^{\prime}\right)$. By $(6), f\left(\nu^{\prime}\right) \geq h\left(\nu^{\prime}\right)$. Thus,

(i) 'there exists $\nu^{\prime}>\mu$ with $g\left(\nu^{\prime}\right)<h\left(\nu^{\prime}\right) \leq f\left(\nu^{\prime}\right)$.

On the other hand, if (3.6) (ii) holds for $f$ and $g$, then by (6),

(ii)' there exists $\nu>\mu$ with $h(\nu) \leq f(\nu)<g(\nu)$.

Note that any $\nu$ satisfying (ii)' lies in $\operatorname{supp}(g)$. Thus, if there exists $\nu$ satisfying (ii)', we may assume that $\nu$ is maximal with this property. Since $g$ $\checkmark h$, there exists $\nu^{\prime}>\nu$ with $g\left(\nu^{\prime}\right)<h\left(\nu^{\prime}\right)$. Since $\nu^{\prime}>\mu$, (6) implies that $\nu^{\prime}$ satisfies (i)'. But since $f \preceq g$, there exists $\nu^{\prime \prime}>\nu^{\prime}$ with $f\left(\nu^{\prime \prime}\right)<g\left(\nu^{\prime \prime}\right)$. Then, (6) implies that $\nu^{\prime \prime}$ satisfies (ii)'. Since $\nu^{\prime \prime}>\nu$, this contradicts the maximality of $\nu$.

Similarly, assuming that $(\mathrm{i})^{\prime}$ holds for some $\nu^{\prime}$ also leads to a contradiction.

If $V$ is an affinization of $\lambda$, define $f_{V} \in \mathscr{F}$ by

$$
f_{V}(\mu)=m_{\mu}(V), \quad\left(\mu \in P^{+}\right)
$$

It is clear that $f_{V}$ depends only on the equivalence class of $V$, and that the map $\mathscr{Q}^{\lambda} \rightarrow \mathscr{F}$ given by $[V] \rightarrow f_{V}$ is injective. Thus, $\preceq$ induces a partial order on $\mathscr{Q}^{\lambda}$, which we also denote by $\leftrightharpoons$. 
Definition 3.8. If $\lambda \in P^{+}$and $[V],[W] \in \mathscr{Q}^{\lambda}$, we write $[V] \preceq[W]$ iff, for all $\mu \in P^{+}$, either,

(i) $m_{\mu}(V) \leq m_{\mu}(W)$, or

(ii) there exists $\nu>\mu$ with $m_{\nu}(V)<m_{\nu}(W)$.

An affinization $V$ of $\lambda$ is minimal if $[V]$ is a minimal element of $\mathscr{Q}^{\lambda}$ for the partial order $\checkmark$, i.e. if $[W] \in \mathscr{Q}^{\lambda}$ and $[W] \preceq[V]$ implies that $[V]=[W]$.

It follows immediately from 3.5 that

Corollary 3.9. For any $\lambda \in P^{+}$, minimal affinizations of $\lambda$ exist.

\section{§4. The Rank 1 Case}

In this section, $\mathrm{g}=s l_{2}(\mathbf{C})$ and $I=\{1\}$.

Definition 4.1. Let $r \in \mathbf{N}, a \in \mathbf{C}^{\times}$. The q-segment $S_{r, a}$ of length $r$ and centre $a$ is the set of non-zero complex numbers $\left\{a q^{-r+1}, a q^{-r+3}, \ldots, a q^{r-1}\right\}$. Two $q^{-}$segments $S_{1}$ and $S_{2}$, of lengths $r_{1}$ and $r_{2}$, are said to be in special position if $S_{1} \cup S_{2}$ is, when suitably ordered, a q-segment of length strictly greater than max $\left\{r_{1}, r_{2}\right\}$; otherwise, $S_{1}$ and $S_{2}$ are said to be in general position.

We recall the main result of $[3]$ :

Theorem 4.2. Let $r \in \mathbf{N}, a \in \mathbf{C}^{\times}$and write

$$
P_{r, a}(u)=\prod_{k=1}^{r}\left(1-a^{-1} q^{r-2 k+1} u\right)
$$

so that the roots of $P_{r, a}$ are the elements of $S_{r, a}$. Then:

(a) $V\left(P_{r, a}\right)$ is irreducible as a representation of $U_{q}\left(s l_{2}\right)$, and has dimension $r+1$

(b) a tensor product

$$
V\left(P_{r_{1}, a_{1}}\right) \otimes V\left(P_{r_{2}, a_{2}}\right) \otimes \cdots \otimes V\left(P_{r_{m}, a_{m}}\right)
$$

where $r_{1}, r_{2}, \ldots, r_{m} \in \mathbb{N}, a_{1}, a_{2}, \ldots a_{m} \in \mathbf{C}^{\times}$, is irreducible as a representation of $U_{q}\left(\widehat{s}_{2}\right)$ iff each pair of q-segments $S_{r_{k}, a_{k}}, S_{r_{l}, a_{l}}$, for $1 \leq_{k}<l \leq m$, is in general position. Moreover, two irreducible tensor products of the form (7) are isomorphic as representation of $U_{q}\left(\hat{s}_{2}\right)$ iff one is obtained from the other by permuting the factors in the tensor product; 
(c) every finite-dimensional irreducible representation of $U_{q}\left(\hat{s}_{2}\right)$ of type 1 is isomorphic to a tensor product of the form (7).

Corollary 4.3. For any $r \in \mathbb{N}, \mathscr{Q}^{r_{1}}$ has a unique minimal element. This element is represented by $V(P)$, where $P$ is any polynomial of degree $r$ whose roots form a q-segment. If $[W] \in \mathscr{Q}^{r \lambda_{1}}$ is not minimal, then $m_{(r-2) \lambda_{1}}(W)>0$.

Proof. The first part is immediate from 3.4 and 4.2 . If $[W] \in \mathscr{Q}^{r \lambda_{1}}$ is not minimal, then by $4.2(\mathrm{c})$,

$$
W \cong V\left(P_{r_{1}, a_{1}}\right) \otimes \cdots \otimes V\left(P_{r_{m}, a_{m}}\right)
$$

where $r_{1}+\cdots+r_{m}=r$ and $m>1$. By 2.1 (e) and the well-known ClebschGordan decomposition for representations of $s_{2}(\mathbb{C})$, the second part of 4.3 follows.

We record the following result here, as it will be needed later. It is an immediate consequence of Proposition 4.9 in [3].

Proposition 4.4. Let $r, s \in \mathbb{N}, a, b \in \mathbb{C}^{\times}$, and let $v, w$ be $U_{q}\left(\widehat{s} l_{2}\right)$ highest weight vectors in $V\left(P_{r, a}\right), V\left(P_{s, b}\right)$, respectively. Then, $W=U_{q}\left(\widehat{s}_{2}\right) .(v \otimes w)$ is a proper $U_{q}\left(\hat{s}_{2}\right)$-subrepresentation of $V=V\left(P_{r, a}\right) \otimes V\left(P_{s, b}\right)$ iff $b / a=q^{r+s-2 p+2}$ for some $0<p \leq \min \{r, s\}$. In that case, $W$ and $V / W$ are irreducible as representation of $U_{q}\left(\hat{s} l_{2}\right)$ and, as representations of $U_{q}\left(s l_{2}\right)$,

$$
\begin{array}{r}
W \cong V\left((r+s) \lambda_{1}\right) \oplus V\left((r+s-2) \lambda_{1}\right) \oplus \cdots \oplus V\left((r+s-2 p+2) \lambda_{1}\right), \\
V / W \cong V\left((r+s-2 p) \lambda_{1}\right) \oplus V\left((r+s-2 p-2) \lambda_{1}\right) \oplus \cdots \oplus V\left(|r-s| \lambda_{1}\right) .
\end{array}
$$

\section{\$5. Classification in the Rank 2 Case}

In this section, $\mathfrak{g}$ is of rank 2 and $I=\{1,2\}$.

Theorem 5.1. Let $\lambda=r_{1} \lambda_{1}+r_{2} \lambda_{2} \in P^{+}$. Then, $\mathscr{Q}^{\lambda}$ has a unique minimal element. This element is represented by $V(\mathbb{P})$, where $\mathbb{P} \in \mathscr{P}^{\lambda}$, iff the following two conditions are satisfied:

(a) for each $i=1,2$, either $P_{i}=1$ or the roots of $P_{i}$ form a $q_{i}$-segment of length $r_{i}$ and centre $a_{i}($ say);

(b) if $P_{1} \neq 1$ and $P_{2} \neq 1$, then

$$
\frac{a_{1}}{a_{2}}=q^{d_{1} r_{1}+d_{2} r_{2}+2 d_{2}-1} \quad \text { or } \quad q^{-\left(d_{1} r_{1}+d_{2} r_{2}+2 d_{1}-1\right)}
$$


The proof of 5.1 will occupy the remainder of this section. If $g$ is of type $A_{2}$, it is proved in [4]. From now on, we assume that $g$ is of type $C_{2}$ or $G_{2}$.

Proof of 5.1 (a). To prove that 5.1 (a) is necessary, we need the following three lemmas.

To state the first lemma, we note that, for each $i=1,2$, there is an algebra homomorphism $U_{q_{i}}\left(\hat{s} l_{2}\right) \rightarrow U_{q}(\hat{\mathrm{g}})$ such that $x_{1, r}^{ \pm} \mapsto x_{i, r}^{ \pm}, k_{1} \mapsto k_{i} h_{1, r} \mapsto h_{\imath, r}$ (this is clear from 1.3). Let $\widehat{U}_{(i)}$ be the image of this map; it is known [1] that $\widehat{U}_{(i)} \cong U_{q_{i}}\left(\widehat{s l}_{2}\right)$. The subalgebra $U_{(i)} \cong U_{q_{t}}\left(s l_{2}\right)$ of $U_{q}(\mathrm{~g})$ is defined similarly. For any $\mu \in P$, let $\mu_{(i)}$ be the restriction of $\mu$ to $\{i\}$. Define $Q_{(i)}=\mathbf{Z} . \alpha_{i}$ and $Q_{(i)}^{+}=\mathbf{Z}_{+} . \alpha_{i}$. Note that $U_{(\mathfrak{})}$ is a Hopf subalgebra of $U_{q}(\mathrm{~g})$, but that $\widehat{U}_{(\mathfrak{})}$ is not a Hopf subalgebra of $U_{q}(\mathrm{~g})$. We denote by $\Delta_{(i)}$ the comultiplication of $\widehat{U}_{(i)}$ and $U_{(i)}$ induced by their isomorphisms with $U_{q_{i}}\left(\widehat{s}_{2}\right)$ and $U_{q_{t}}\left(s l_{2}\right)$, respectively.

Now let $\lambda \in P^{+}$, let $\mathbf{P} \in \mathscr{P}^{\lambda}$, and let $M$ be a highest weight representation of $U_{q}(\hat{\mathfrak{g}})$ with highest weight $\mathbf{P}$ and highest weight vector $m$. Let $M_{(i)}=\widehat{U}_{(i)} m$. Then, it follows from 1.4 that

$$
M_{(i)}=\bigoplus_{\eta \in Q_{(z)}^{+}} M_{\lambda-\eta}
$$

Similarly, let $\mu \in P^{+}, \mathbf{Q} \in \mathscr{P}^{\mu}$, let $N$ be a highest weight representation of $U_{q}(\hat{\mathfrak{g}})$ with highest weight $\mathbf{Q}$ and highest weight vector $n$, and let $N_{(i)}=\widehat{U}_{(i)} . n$. Then, we have

$$
M_{(i)} \otimes N_{(i)}=\bigoplus_{\eta \in Q_{(i)}^{+}}(M \otimes N)_{\lambda+\mu-\eta} .
$$

Indeed, it is obvious that the left-hand side of (9) is contained in the right-hand side. On the other hand,

$$
(M \otimes N)_{\lambda+\mu-\eta}=\bigoplus_{\eta^{\prime}, \eta^{\prime \prime}} M_{\lambda-\eta^{\prime}} \otimes N_{\mu-\eta^{\prime \prime}}
$$

where the sum is over those $\eta^{\prime}, \eta^{\prime \prime} \in Q^{+}$such that $\eta^{\prime}+\eta^{\prime \prime}=\eta$. But, since $\eta \in$ $Q_{(i)}^{+}$, this clearly forces $\eta^{\prime}, \eta^{\prime \prime} \in Q_{(i)}^{+}$, so by (8), $(M \otimes N)_{\lambda+\mu-\eta} \subseteq M_{(i)} \otimes N_{(i)}$. This proves (9).

Now, $M_{(i)} \otimes N_{(\imath)}$ admits an obvious action of $\widehat{U}_{(i)}$ by using $\Delta_{(\imath)}$; we denote this representation by $M_{(i)} \otimes_{(i)} N_{(\imath)}$. On the other hand, for weight reasons, the action of the $\Delta\left(x_{i, r}^{ \pm}\right), \Delta\left(\phi_{i, r}^{ \pm}\right)$, for all $r \in \mathbf{Z}$, obviously preserves $\oplus_{\eta \in Q_{(t)}^{+}}(M \otimes N)$ 
$\lambda+\mu-\eta$. This gives another representation of $\widehat{U}_{(i)}$ on $M_{(i)} \otimes N_{(i)}$, using $\Delta$, which we denote by $M_{(i)} \otimes N_{(i)}$.

Lemma 5.2. (a) For $i=1,2$,

$$
\Delta\left(x_{i, \pm 1}^{ \pm}\right)-\Delta_{(i)}\left(x_{i, \pm 1}^{ \pm}\right) \in \bigoplus_{\eta^{\prime}, \eta^{\prime \prime}} U_{q}(\hat{\mathfrak{g}})_{\eta^{\prime}} \otimes U_{q}(\hat{\mathfrak{g}})_{\eta^{\prime \prime}}
$$

where the sum is over those $\eta^{\prime}, \eta^{\prime \prime} \in Q \backslash Q_{(i)}$ such that $\eta^{\prime}+\eta^{\prime \prime}= \pm \alpha_{i}$, and

$$
U_{q}(\hat{\mathfrak{g}})_{\eta}=\left\{u \in U_{q}(\hat{\mathfrak{g}}) \mid k_{j} u k_{j}^{-1}=q_{j}^{\eta(j)} u \quad \text { for all } \quad j \in I\right\}
$$

(b) The identity map $M_{(i)} \otimes_{(i)} N_{(i)} \rightarrow M_{(i)} \otimes N_{(i)}$ is an isomorphism of representations of $\widehat{U}_{(i)}$.

Lemma 5.3. Let $\mathbb{P} \in \mathscr{P}$ and let $v_{\mathbf{P}}$ be a highest weight vector of $V(\mathbb{P})$. Then, for each $i=1,2, U_{(i)} . v_{\mathbf{p}}$ is an irreducible representation of $U_{q_{1}}\left(\hat{s}_{2}\right)$ with highest weight $P_{i}$. Moreover, if $\mu=\lambda-s \alpha_{i} \in P$ for some $s \in \mathbb{Z}_{+}$,

$$
m_{\mu}(V(\mathbf{P}))=m_{\mu(t)}\left(\widehat{U}_{(i)} \cdot v_{\mathbf{p}}\right)
$$

Lemma 5.4. Let $\lambda \in P^{+}, \mathbb{P} \in \mathscr{P}^{\lambda}$. $>0$.

(a) If $P_{i} \neq 1$ and the roots of $P_{i}$ do not form a $q_{i}$-segment, then $m_{\lambda-\alpha_{i}}(V(\mathbb{P}))$

(b) If $P_{i}=1$, or if $P_{i} \neq 1$ and the roots of $P_{i}$ form a $q_{i}$-segment, then, for all $r>$ $0, m_{\lambda-r \alpha_{i}}(V(\mathbb{P}))=0$.

Assuming these lemmas, suppose that $V(\mathbf{P})$ is minimal but that, for some $i$ $\in I, P_{i} \neq 1$ and the roots of $P_{i}$ do not form a $q_{i}{ }^{-}$segment. Let $\mathbb{Q}=\left(Q_{j}\right)_{j \in I} \in \mathscr{P}^{\lambda}$ be such that, for $j=1,2$, if $Q_{j} \neq 1$ the roots of $Q_{j}$ form a $q_{j}$-segment. We claim that $[V(\mathbf{Q})] \prec[V(\mathbb{P})]$.

Let $\mu \in P^{+}$be such that $m_{\mu}(V(\mathbb{Q}))>0, \mu \neq \lambda$. By $5.3(\mathrm{~b}), \mu=\lambda-s_{1} \alpha_{1}-$ $s_{2} \alpha_{2}$, where $s_{1}, s_{2}>0$. Hence, $\mu<\lambda-\alpha_{i}$, and, by $5.4(\mathrm{a})$,

$$
m_{\lambda-\alpha_{\imath}}(V(\mathbb{P}))>0, m_{\lambda-\alpha_{\imath}}(V(\mathbf{Q}))=0,
$$

so $\mu$ satisfies 3.8 (ii). This proves our claim, and hence also that 5.1 (a) is necessary.

Proof of 5.2. (a) This follows in a straightforward manner from [1].

(b) The map obviously commutes with the action of $U_{(i)}$. From 1.3 , it fol- 
lows that $\widehat{U}_{(i)}$ is generated as an algebra by the elements of $U_{(i)}$, the $x_{i, r}^{ \pm}$for $r=$ \pm 1 , and the $c^{ \pm 1 / 2}$. Since $c^{1 / 2}$ acts as the identity on $M$ and $N$, it suffices to prove that, for all $m^{\prime} \in M_{(i)}, n^{\prime} \in N_{(i)}, r= \pm 1$,

$$
\Delta\left(x_{i, r}^{ \pm}\right) .\left(m^{\prime} \otimes n^{\prime}\right)-\left(\rho_{(i)} \otimes \rho_{(i)}\right)\left(\Delta_{(i)}\left(x_{i, r}^{ \pm}\right)\right) .\left(m^{\prime} \otimes n^{\prime}\right)=0
$$

The left-hand side of (10) obviously belongs to $M_{(i)} \otimes N_{(t)}$, since both terms involved do. On the other hand, by part (a), the left-hand side also belongs to

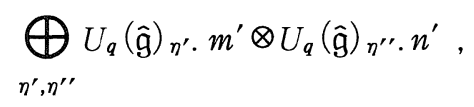

where the sum is over those $\eta^{\prime}, \eta^{\prime \prime} \in Q \backslash Q_{(\imath)}$ such that $\eta^{\prime}+\eta^{\prime \prime}= \pm \alpha_{\imath}$. We may assume that $m^{\prime} \in M_{\lambda-\xi^{\prime}}, n^{\prime} \in N_{\mu-\xi^{\prime \prime}}$, where $\xi^{\prime}, \xi^{\prime \prime} \in Q_{(i)}^{+}$. Then, the weight of the first factor in a typical non-zero term in the above sum is $\lambda-\xi^{\prime}+\eta^{\prime}$. On the other hand, by (8), its weight must be of the form $\lambda-\eta$ for some $\eta \in Q_{(i)}^{+}$. Thus,

$$
\eta^{\prime}=\xi^{\prime}-\eta
$$

But this is impossible, since $\xi^{\prime}-\eta \in Q_{(i)}^{+}$but $\eta^{\prime} \notin Q_{(\imath)}^{+}$. Hence, the left-hand side of (10) is zero.

Proof of 5.3. Suppose that $\widehat{U}_{(\imath)} . v_{\mathbf{p}}$ is reducible. Then, by $2.2(\mathrm{~b})$, there exists $v \in \widehat{U}_{(i)}$. $v_{\mathbf{P}}$, not a multiple of $v_{\mathbf{P}}$, such that $v$ is annihilated by $x_{i, r}^{+}$for all $r$ $\in \mathbf{Z}$ and is an eigenvector of $k_{\imath}$. It is easy to see from the relations in 1.3 that the set of such vectors $v$ is preserved by the action of the $\phi_{j, s}^{ \pm}$, for all $j \in I, s \in \mathbb{Z}$. Hence, we may assume that

$$
\phi_{j, s .}^{ \pm} v=\Phi_{j, s}^{ \pm} v \quad(j \in I, s \in \mathbb{Z})
$$

for some $\Phi_{j, s}^{ \pm} \in \mathbb{C}$. In particular, $v$ is a common eigenvector of $k_{1}$ and $k_{2}$, and since $v \in \widehat{U}_{(\imath)} . v \mathbf{P}$, its weight is clearly of the form $\lambda-t \alpha$ for some $t \in \mathbb{Z}$. Then, $x_{j, r}^{+} . v=0$ for $j \neq i$ as well. This shows that $v$ is a $U_{q}(\hat{\mathfrak{g}})$-highest weight vector, which contradicts the irreducibility of $V(\mathbb{P})$.

For the second statement, observe from 1.4 that

$$
\widehat{U}_{(i) .} v_{\mathbf{P}}=\bigoplus_{r \in \mathbf{Z}_{+}} V(\mathbb{P})_{\lambda-r a_{i}}
$$


Hence $x_{j, 0}^{+} . \widehat{U}_{(i)}, v_{\mathbf{P}}=0$ if $j \neq i . \quad$ The result now follows.

Proof of 5.4. (a) By 5.3, $\widehat{U}_{(\mathfrak{t})} . v_{\mathbf{P}}$ is irreducible as a representation of $U_{q_{\mathrm{t}}}\left(\hat{s} l_{2}\right)$. By 4.3, there exists $0 \neq v \in \widehat{U}_{(i)} . v \mathbf{P} \cap V(\mathbf{P})_{\lambda-\alpha_{i}}$ such that $x_{i, 0}^{+} v=0$. Clearly then $x_{j, 0}^{+} . v=0$ for $j \neq i$ so $m_{\lambda-\alpha_{1}}(V(\mathbf{P}))>0$.

(b) If $P_{i}=1$, the statement is clear, since then $\lambda-r \alpha_{i} \notin P^{+}$. Let $P_{i} \neq 1$ be such that the roots of $P_{i}$ form a $q_{i}$-segment. If $m_{\lambda-r \alpha_{i}}(V(\mathbf{P})) \neq 0$, then, by 1.4 , we see that there exists $0 \neq v \in V(\mathbf{P})_{\lambda-r \alpha_{i}} \cap \widehat{U}_{(i)} . v_{\mathbf{P}}$ such that $x_{i, 0}^{+} . v=0$. But, by 5.3 and $4.2(\mathrm{a}), \widehat{U}_{(i)} \cdot v \mathbf{P}$ is irreducible as a representation of $U_{q_{\mathbf{t}}}\left(s l_{2}\right)$, so this is impossible.

Before showing that 5.1 (b) holds, we show that if one of $P_{1}, P_{2}$ is equal to 1 , say $P_{1}$ without loss, and the roots of $P_{2}$ form a $q_{2}$-segment, then $[V(\mathbf{P})]$ is minimal. For, suppose $\mathbf{Q} \in \mathscr{P}^{\lambda}$ is such that $[V(\mathbf{Q})]$ is minimal and $[V(\mathbf{Q})]$ $\checkmark[V(\mathbf{P})]$. Then $Q_{1}=1$, and since 5.1 (a) is necessary, the roots of $Q_{2}$ form a $q_{2}$-segment. But then, for some $t \in \mathbf{C}^{\times}, \tau_{t}^{*}(V(\mathbf{Q})) \cong V(\mathbf{P})$ by 3.4 , so $[V(\mathbf{Q})]=$ $[V(\mathbf{P})]$ and $[V(\mathbf{P})]$ is minimal. This establishes 5.1 when $r_{i}=0$ for some $i=$ 1,2 .

From now on, we assume that $P_{i} \neq 1, i=1,2$, and that the roots of $P_{i}$ form a $q_{i}$-segment with centre $a_{i} \in \mathbb{C}^{\times}, i=1,2$. To complete the proof of 5.1 , we need the following two results.

Proposition 5.5. Let $g$ be of type $C_{2}$ or $G_{2}$, and let $\mu=r_{1} \lambda_{1}+r_{2} \lambda_{2} \in P^{+}$. Assume that, if $Q_{i} \neq 1$, the roots of $Q_{i}$ form a $q_{i}$-segment of length $r_{i}$ and centre $b_{i}$.

(a) If $Q_{i}=1$ for some $i=1,2$, then, for all $s_{i}>0$,

$$
m_{\mu-s_{\imath} \alpha_{i}}(V(\mathbf{Q}))=0, m_{\mu-\alpha_{1}-\alpha_{2}}(V(\mathbf{Q}))=0 .
$$

(b) Assume that $Q_{i} \neq 1, i=1,2$. Let $M$ be a highest weight representation of $U_{q}(\hat{\mathfrak{g}})$ with highest weight $\mathbf{Q} \in \mathscr{P}^{\mu}$ such that

$$
m_{\mu-\alpha_{1}-\alpha_{2}}(M)=0, \quad m_{\mu-\alpha_{1}}(M)=0,
$$

for $i=1,2$. Then,

$$
\frac{b_{1}}{b_{2}}=q^{d_{1} r_{1}+d_{2} r_{2}+2 d_{2}-1} \quad \text { or } \quad q^{-\left(d_{1} r_{1}+d_{2} r_{2}+2 d_{1}-1\right)}
$$

(c) Assume that $Q_{i} \neq 1$ and define $Q^{(i)} \in \mathscr{P P}^{r_{1} \lambda_{s}}$ as follows:

$$
Q_{j}^{(\imath)}= \begin{cases}Q_{i} & \text { if } i=j \\ 1 & \text { if } i \neq j\end{cases}
$$


Let $v_{\imath}$ be a $U_{q}(\hat{\mathfrak{g}})$-highest weight vector in $V\left(\mathbf{Q}^{(i)}\right)$, and let $M=U_{q}(\hat{\mathfrak{g}}) .\left(v_{i} \otimes v_{j}\right) \subset$ $V\left(\mathbf{Q}^{(i)}\right) \otimes V\left(\mathbf{Q}^{(j)}\right), i \neq j . \quad$ Then

$$
m_{\lambda-\alpha_{1}-\alpha_{2}}(M)=0 \quad \text { iff } \frac{b_{i}}{b_{j}}=q^{-\left(d_{1} r_{1}+d_{2} r_{2}+2 d_{1}-1\right)} .
$$

If $\rho: U_{q}(\hat{\mathfrak{g}}) \rightarrow E n d(V)$ is a representation, denote the representation $\rho \circ \widehat{\omega}$ by $\widehat{\omega}^{*}(V)$.

Proposition 5.6. Let $\mathrm{g}$ be of type $C_{2}$ or $G_{2}$. Let $\mu=r_{1} \lambda_{1}+r_{2} \lambda_{2} \in P^{+}$, let $\mathbf{Q}$ $=\left(Q_{i}\right)_{i \in I} \in \mathscr{g}^{\mu}$, and let

$$
Q_{i}(u)=\prod_{r=1}^{r_{i}}\left(1-a_{i, r}^{-1} u\right) \quad(i=1,2)
$$

Define polynomials $\bar{Q}_{i}(u)$ by

$$
\bar{Q}_{\imath}(u)=\prod_{r=1}^{r_{i}}\left(1-q_{i}^{2} a_{i, r} u\right)
$$

Then, $V(\overline{\mathbf{Q}}) \cong \tau_{t}^{*} \widehat{\omega}^{*}(V(\mathbf{Q}))$ for some $t \in \mathbf{C}^{\times}$.

Assuming these propositions, we complete the proof of 5.1 as follows. Suppose that $[V(\mathbf{P})]$ is minimal but that $a_{1} / a_{2}$ has neither of the values stated in $5.1(\mathrm{~b})$. By $5.4(\mathrm{~b}), m_{\lambda-\alpha_{\mathbf{t}}}(V(\mathbf{P}))=0$, so by $5.5(\mathrm{~b})$,

$$
m_{\lambda-\alpha_{1}-\alpha_{2}}(V(\mathbf{P}))>0
$$

Choose $\mathbf{Q}=\left(Q_{i}\right)_{i \in I} \in \mathscr{P}^{\lambda}$ such that the roots of $Q_{\imath}$ form a $q_{i}$-segment with centre $b_{i}$, where $b_{1} / b_{2}$ has one of the values in $5.1(\mathrm{~b})$. By $5.5(\mathrm{c})$,

$$
m_{\lambda-\alpha_{1}-\alpha_{2}}(V(\mathbf{Q}))=0
$$

Hence, $[V(\mathbf{Q})] \neq[V(\mathbf{P})]$. If $m_{\mu}(V((\mathbf{Q}))>0, \mu \neq \lambda$, then by $5.4(\mathrm{~b}), \mu=\lambda-$ $s_{1} \alpha_{1}-s_{2} \alpha_{2}$ where $s_{1}, s_{2}>0$, and since $\mu \neq \lambda-\alpha_{1}-\alpha_{2}$, we have $\mu<\lambda-\alpha_{1}-\alpha_{2}$. Hence $\mu$ satisfies 3.8 (ii), and $[V(\mathbf{Q})] \prec[V(\mathbf{P})]$, contradicting minimality of $[V(\mathbf{P})]$.

Conversely, suppose $\mathbf{P}$ is such that conditions 5.1 (a) and 5.1 (b) are both satisfied. Choose $\mathbf{Q}=\left(Q_{\imath}\right)_{i \in I} \in \mathscr{P}^{\lambda}$ such that $[V(\mathbf{Q})]$ is minimal and $[V(\mathbf{Q})]$ $\checkmark[V(\mathbf{P})]$. Since conditions $5.1(\mathrm{a})$ and (b) are necessary, the roots of $Q_{i}$ 
must form a $q_{i}$-segment with centre $b_{i}$, say, where $b_{1} / b_{2}$ also has one of the values in 5.1 (b). If $a_{1} / a_{2}=b_{1} / b_{2}$, then, by $3.4, V(\mathbb{Q}) \cong \tau_{t}^{*}(V(\mathbb{P}))$ for some $t \in$ $\mathbb{C}^{\times}$, and then $[V(\mathbf{Q})]=[V(\mathbb{P})]$. On the other hand, if $a_{1} / a_{2}=q^{d_{1} r_{1}+d_{2} r_{2}+2 d_{2}-1}$ (resp. $\left.q^{-\left(d_{1} r_{1}+d_{2} r_{2}+2 d_{1}-1\right)}\right)$ and $\left.b_{1} / b_{2}=q^{-\left(d_{1} r_{1}+d_{2} r_{2}+2 d_{1}-1\right.}\right)$ (resp. $q^{d_{1} r_{1}+d_{2} r_{2}+2 d_{2}-1}$ ), then by $5.6, V(\mathbb{Q}) \cong \widehat{\omega}^{*}(V(\mathbb{P}))$, and again $[V(\mathbb{Q})]=[V(\mathbb{P})]$. In both cases, $[V(\mathbb{P})]$ is minimal.

We continue to assume 5.5 and prove 5.6 .

Proof of 5.6. We first reduce to the case when $V(\mathbb{Q})$ fundamental. By 2.6, $V(\mathbb{Q})$ is isomorphic to the unique irreducible subquotient of

$$
\bigotimes \bigotimes_{i \in I}^{r_{i}} V\left(\lambda_{i}, a_{i, r}\right)
$$

which contains a $U_{q}(\mathfrak{g})$-subrepresentation isomorphic to $V(\mu)$, and hence $\widehat{\omega}^{*}(V(\mathbb{Q}))$ is isomorphic to the unique irreducible subquotient of

$$
\widehat{\omega}^{*}\left(\bigotimes_{i \in I} \bigotimes_{r=1}^{r_{t}} V\left(\lambda_{i}, a_{i, r}\right)\right) \cong \bigotimes_{i \in I} \bigotimes_{r=1}^{r_{1}} \hat{\omega}^{*}\left(V\left(\lambda_{i}, a_{i, r}\right)\right)
$$

which contains a $U_{q}(\mathrm{~g})$-subrepresentation isomorphic to $V$ (the order of the factors in the tensor product on the right-hand side of (13) is the reverse of that on the left-hand side). It is clear that the unique such quotient of

$$
\bigotimes \bigotimes_{r \in I}^{r_{i}} V\left(\lambda_{i}, t a_{i, r}^{-1}\right)
$$

is isomorphic to $\left(\tau_{t}^{*}\right)^{-1}(V(\overline{\mathrm{Q}}))$.

To prove the result in the fundamental case, note that, by 3.3 ,

$$
\widehat{\omega}^{*}\left(V\left(\lambda_{i}, a_{\imath}\right)\right) \cong V\left(\lambda_{i}, \overline{a_{i}}\right), \quad(i=1,2),
$$

for some $\overline{a_{i}} \in \mathbb{C}^{\times}$(not necessarily the complex conjugate of $a_{i}$ ). Assume that $a_{1} / a_{2}=q^{-\left(3 d_{1}+d_{2}-1\right)}$. By 5.5 , if $v_{\imath}$ is a $U_{q}(\hat{\mathfrak{g}})$-highest weight vector in $V\left(\lambda_{i}, a_{i}\right)$, and $M=U_{q}(\hat{\mathfrak{g}}) .\left(v_{1} \otimes v_{2}\right)$, then $m_{\lambda_{1}+\lambda_{2}-\alpha_{1}-\alpha_{2}}(M)=0 . \quad$ Clearly, $M^{\prime}=U_{q}(\hat{\mathfrak{g}}) .\left(v_{2} \otimes v_{1}\right)$ $\subseteq \widehat{\omega}^{*}(M)$, hence $m_{\lambda_{1}+\lambda_{2}-\alpha_{1}-\alpha_{2}}\left(M^{\prime}\right)=0$. By 5.5 again,

$$
\frac{\overline{a_{2}}}{\overline{a_{1}}}=q^{-\left(d_{1}+3 d_{2}-1\right)} .
$$


Hence,

$$
q_{1}^{2} a_{1} \overline{a_{1}}=q_{2}^{2} a_{2} \overline{a_{2}},
$$

so the result follows from 3.4.

Proof of 5.5 (a). We assume that $d_{2}=1$.

The fact that $m_{\mu-s_{1} \alpha_{i}}(V(\mathbf{Q}))=0$ follows from 5.4 (b). If $Q_{1}=1$ it is enough to notice that $\mu-\alpha_{1}-\alpha_{2} \notin P^{+}$.

If $Q_{2}=1$, we must consider separately the cases when $g$ is of type $C_{2}$ or $G_{2}$. Let $v_{\mathbf{Q}}$ be a $U_{q}(\hat{\mathrm{g}})$-highest weight vector of $V(\mathbf{Q})$.

If $g$ is of type $C_{2}$, then $x_{0}^{+} . v_{\mathbf{Q}}$ has weight $r_{1} \lambda_{1}-\alpha_{1}-2 \alpha_{2}$ which is Weyl group conjugate to $r_{1} \lambda_{1}-\alpha_{1} \in P^{+}$if $r_{1}>1$ and to $\lambda_{1}$ if $r_{1}=1$. Hence, if $m_{\nu}(V(\mathbf{Q}))>0$ and $x_{0}^{+}$. $v_{\mathbf{Q}}$ has a non-zero component in a $U_{q}(\mathfrak{g})$-subrepresentation of $V(\mathbf{Q})$ of highest weight $\nu$, then $\nu=\lambda_{1}$ if $r_{1}=1$ and if $r>1$, then $\nu=r_{1} \lambda_{1}$ or $r_{1} \lambda_{1}$ $-\alpha_{1}$. But, $m_{r_{1} \lambda_{1}-\alpha_{1}}(V(\mathbf{Q}))=0$ by $5.4(\mathrm{~b})$, so $x_{0}^{+} . v_{\mathbf{Q}} \in U_{q}(\mathrm{~g}) . v_{\mathbf{Q}} \cong V\left(r_{1} \lambda_{1}\right)$. Similarly, if $v_{\mathbf{Q}}^{*}$ is a $U_{q}(\hat{\mathfrak{g}})$-lowest weight vector of $V(\mathbf{Q})$, then $x_{0}^{-} \cdot v_{\mathbf{Q}}^{*} \in U_{q}(\mathfrak{g}) . v_{\mathbf{Q}}^{*}$ $=U_{q}(\mathfrak{g}) . v_{\mathbf{Q}}$. It follows that $x_{0}^{ \pm}$preserve $U_{q}(\mathfrak{g}) . v_{\mathbf{Q}}$, and hence that $U_{q}(\mathfrak{g}) . v_{\mathbf{Q}}$ is a $U_{q}(\hat{\mathfrak{g}})$-subrepresentation of $V(\mathbf{Q})$. This not only proves $5.5(\mathrm{a})$, but the following stronger result:

Proposition 5.7. If $\mathrm{g}$ is of type $C_{2}$, where $d_{2}=1$, and if $r \in \mathbf{N}$, then $r \lambda_{1}$ has an affinization which is irreducible as a representation of $U_{q}(\mathfrak{g})$ (this necessarily represents the unique minimal element of $\mathscr{Q}^{r \lambda_{1}}$ ).

If $\mathfrak{g}$ is of type $G_{2}$ then $x_{2}^{+} x_{0}^{+} . v_{\mathbf{Q}}=0$ since $\left[x_{2}^{+}, x_{0}^{+}\right]=0$. Assume for a contradiction that $x_{0}^{+} . v_{\mathbf{Q}}$ has a non-zero component $w$ in a $U_{q}(\mathrm{~g})$-subrepresentation of $V(\mathbf{Q})$ isomorphic to $V\left(r_{1} \lambda_{1}-\alpha_{1}-\alpha_{2}\right)$. Since $x_{2}^{+} . w \in V\left(r_{1} \lambda_{1}-\alpha_{1}-\alpha_{2}\right)$, the preceding comment shows that in fact $x_{2}^{+} . w=0$. Now $w$ is an element of weight $\left(r_{1}-1\right) \lambda_{1}$ in the $U_{q}(\mathrm{~g})$-irreducible module $V\left(r_{1} \lambda_{1}-\alpha_{1}-\alpha_{2}\right)$ and hence cannot be $U_{q}(\mathfrak{g})$-highest weight, thus forcing $x_{1}^{+} . w \neq 0$. This means that $\left(r_{1}-1\right) \lambda_{1}+$ $\alpha_{1}$, and hence also its Weyl group conjugate $\left(r_{1}-1\right) \lambda_{1}+\alpha_{1}+3 \alpha_{2}$, is a weight of $V\left(r_{1} \lambda_{1}-\alpha_{1}-\alpha_{2}\right)$. This is impossible because $\left(r_{1}-1\right) \lambda_{1}+\alpha_{1}+3 \alpha_{2}>\gamma_{1} \lambda_{1}-\alpha_{1}-$ $\alpha_{2}$.

The proof of $5.5(\mathrm{a})$ is now complete.

We assume from now on that $Q_{\imath} \neq 1, i=1,2$. To prove (b) and (c) we shall need the following result.

Lemma 5.8. Let $M$ be a highest weight $U_{q}(\hat{\mathfrak{g}})$-module with highest weight $\mathbf{Q} \in \mathscr{P}^{\mu}, \mu=r_{1} \lambda_{1}+r_{2} \lambda_{2}$, and highest weight vector $m$. Assume that $m_{\mu-\alpha_{1}}(M)=0$. 
Then,

(a) for $i=1,2, r \in \mathbb{Z}$,

$$
h_{i, r .} m=H_{i, r} m, x_{i, r}^{-} m=X_{i, r} x_{i, 0}^{-} . m
$$

for some $H_{i, r}, X_{i, r} \in \mathbb{C}$. If, in addition, the roots of $Q_{i}$ form a $q_{i}$-segment with centre $b_{i}$, then

$$
H_{i, 1}=q_{\imath}^{-1} b_{i}^{-1}\left[r_{i}\right]_{q_{i}}, \quad X_{i, 1}=b_{i}^{-1} q_{i}^{r_{1}-1}
$$

(b) Let $W \subseteq M$ be the linear span of $\left\{x_{1}^{-} x_{2}^{-}, m, x_{2}^{-} x_{1}^{-} . m\right\}$. Then, the following are equivalent:

(i ) $x_{1,1}^{-} x_{2,0 .}^{-} m \in W$;

(ii) $x_{i, r}^{\bar{r}} x_{j, s .} . m \in W$ for all $i \neq j \in\{1,2\}, r, s \in \mathbb{Z}$;

(iii) $m_{\mu-\alpha_{1}-\alpha_{2}}(M)=0$.

We assume 5.8 and complete the proof of 5.5 .

Proof of $5.5(\mathrm{~b})$. Suppose that (11) is satisfied. By $5.8(\mathrm{~b})$, we can write

$$
x_{1,1}^{-} x_{2,0}^{-} . m=C x_{1,0}^{-} x_{2,0}^{-} . m+D x_{2,0}^{-} x_{1,0}^{-} . m,
$$

where $C, D \in \mathbf{C}$. Applying $x_{1,0}^{+}, x_{2,0}^{+}$and $x_{2,1}^{+}$, respectively, to both sides of this equation, and using 5.8 (a) and the relations in 1.3 , we find the following system of equations for $C, D$ :

$$
\begin{gathered}
q_{1}^{-a_{12}}\left(b_{1}^{-1} q_{1}^{\gamma_{1}-1}\left[r_{1}\right]_{q_{1}}-\left[a_{12}\right]_{q_{1}} q_{1}^{\gamma_{1}} b_{2}^{-1} q_{2}^{\gamma_{2}-1}\right)=C\left[r_{1}-a_{12}\right]_{q_{1}}+D\left[r_{1}\right]_{q_{1}}, \\
{\left[r_{2}\right]_{q_{2}} b_{1}^{-1} q_{1}^{\gamma_{1}-1}=C\left[r_{2}\right]_{q_{2}}+D\left[r_{2}-a_{21}\right]_{q_{2}},} \\
b_{1}^{-1} b_{2}^{-1} q_{2}^{\gamma_{2}-1}\left[r_{2}\right]_{q_{2}} q_{1}^{\gamma_{1}-1}= \\
C b_{2}^{-1} q_{2}^{\gamma_{2}-1}\left[r_{2}\right]_{q_{2}}+ \\
D q_{2}^{-a_{21}}\left(b_{2}^{-1} q_{2}^{\gamma_{2}-1}\left[r_{2}\right]_{q_{2}}-\left[a_{21}\right]_{q_{2}} q_{2}^{\gamma_{2}} b_{1}^{-1} q_{1}^{\gamma_{1}-1}\right) .
\end{gathered}
$$

A straightforward calculation shows that these equations are consistent only if (12) holds.

Proof of 5.5 (c). We prove this when $g$ is of type $C_{2}$; the $G_{2}$ case is similar. By $5.4(\mathrm{~b})$, we know that, for all $i, j, m_{\mu-\alpha j}\left(V\left(\mathbf{Q}^{(2)}\right)\right)=0$. Hence, $m_{\mu-\alpha i}(M)=0$ for $i=1,2$ and, by 5.8 , proving that $m_{\mu-\alpha_{1}-\alpha_{2}}(M)=0$ is equivalent to proving that $\left[\overline{x_{j, 0}}, x_{\bar{i}, 1}\right]_{q \cdot}\left(v_{i} \otimes v_{j}\right)=\left(q x_{\overline{j, 0}}^{\overline{x_{i, 1}}}-q^{-1} x_{\overline{i, 1}} x_{\overline{j, 0}}\right) .\left(v_{\imath} \otimes v_{j}\right)$ is a linear combination of the following two elements: 


$$
\begin{aligned}
& x_{\overline{i, 0}}^{\bar{x}} \overline{x_{j, 0}}\left(v_{i} \otimes v_{j}\right)=\overline{x_{i, 0}}-v_{i} \otimes \overline{x_{j, 0}} \cdot v_{j}+q_{\imath}^{-r_{i}} v_{i} \otimes \overline{x_{i, 0}} \overline{x_{j, 0} .} v_{j}, \\
& \overline{x_{j, 0}} \overline{x_{i, 0}}\left(v_{i} \otimes v_{j}\right)=\overline{x_{j, 0}} \overline{x_{i, 0}} . v_{i} \otimes v_{j}+q_{i}^{-1} \overline{x_{i, 0}} \cdot v_{i} \otimes \overline{x_{j, 0}} . v_{j} .
\end{aligned}
$$

Note that, by the isomorphism $f$ in 1.3 , we have $x_{0}^{+}=\left[x_{2,0}^{-}\left[x_{2,0}^{-}, x_{1,1}^{-}\right]_{q}\right]$ $\left(k_{1} k_{2}^{2}\right)^{-1}$, from which one deduces that

$$
\left[x_{2}^{+}, x_{0}^{+}\right]=(-1)^{\delta_{t, 2}}\left(q^{2}-q^{-2}\right)\left[\overline{x_{j, 0}}, \overline{x_{i, 1}^{-}}\right]_{q}\left(k_{1} k_{2}\right)^{-1} .
$$

Using 1.3 and $5.8(\mathrm{a})$, one finds that

$$
\begin{gathered}
{\left[x_{2}^{+}, x_{0}^{+}\right]\left(v_{\imath} \otimes v_{j}\right)=\left[x_{2}^{+}, x_{0}^{+}\right] . v_{\imath} \otimes k_{2} k_{0} . v_{j}+v_{i} \otimes\left[x_{2}^{+}, x_{0}^{+}\right] . v_{j}} \\
=(-1)^{\delta_{t, 2}}\left(q^{2}-q^{-2}\right)\left(b_{\imath}^{-1} q^{-d_{\imath}-d, r,} x_{j, 0}^{-} x_{\imath, 0}^{-} v_{\imath} \otimes v_{j}-b_{j}^{-1} q^{-1} v_{i} \otimes x_{i, 0}^{-} x_{j, 0}^{-} \cdot v_{j}\right) .
\end{gathered}
$$

It is easy to see that this element is a linear combination of the elements in (14) and (15) if and only if

$$
\frac{b_{i}}{b_{j}}=q^{-\left(d_{1} r_{1}+d_{2} r_{2}+2 d_{t}-1\right)} .
$$

Finally, we give the

Proof of 5.8. (a) That $m$ is a common eigenvector of the $h_{t, r}$ is a consequence of the fact that $m$ is the highest weight vector of $M$ and of the relation between the $\phi_{i, r}^{ \pm}$and the $h_{\imath, r}$ given in 1.3. If the roots of $Q_{i}$ form a $q_{i}$-segment, then, by using (5), it follows immediately that the eigenvalue of $h_{t, 1}$ is as given. To see that $x_{\imath, r}^{-} . m$ is a multiple of $x_{\imath, 0}^{-} . m$, it suffices to note that, in view of the relations in $1.3, x_{i, 0}^{+}$kills a suitable linear combination of $x_{\imath, r}^{-} . m$ and $x_{i, 0}^{-} . m$.

(b) By 5.8 (a), to prove that (i) implies (ii), it suffices to prove that $\overline{x_{i, r}} \overline{x_{j, 0}} . m$ $\in W$ for all $r \in \mathbf{Z}, i \neq j$. By the following relation in $U_{q}(\hat{\mathfrak{g}})$, and an obvious induction on $r$, we may assume that $i=1, j=2$ :

$$
x_{2, r}^{-} x_{1, s}^{-}-q^{-a_{21}} x_{1, s}^{-} x_{2, r}^{-}=q^{-a_{21}} x_{2, r-1}^{-} x_{1, s+1}^{-}-x_{1, s+1}^{-} x_{2, r-1}^{-} .
$$

Since we are given that $\overline{x_{1,1}} x_{2,0}^{-} . m \in W$, we are reduced to proving the following statement:

$$
\text { if } x_{1, r}^{-} x_{2,0}^{-} m \in W \text { for some } r \in \mathbf{Z} \text {, then } x_{1, r \pm 1}^{-} x_{2,0}^{-} m \in W \text {. }
$$

To prove (16), note first that the relation 


$$
\left[h_{\imath, r}, x_{j, s}^{-}\right]=-\frac{1}{r}\left[r a_{i j}\right]_{q^{\prime}} c^{|r| / 2} x_{j, r+s}^{-}
$$

in $U_{q}(\hat{\mathfrak{g}})$, and the fact that $c^{1 / 2}$ acts as the identity on $M$, imply that there exist elements $H_{r} \in U_{q}(\hat{\mathfrak{g}}), r \in \mathbb{Z}$, which are linear combinations of $h_{i, r}, i=1,2$, such that, for all $m^{\prime} \in M, r, s \in \mathbb{Z}$,

$$
\left[H_{s}, x_{i, r}^{-}\right] . m^{\prime}=\delta_{i 1} x_{i, r+s}^{-} \cdot m^{\prime} .
$$

Now, (16) will follow if we prove that $H_{ \pm 1} . W \subseteq W$. For $H_{1}$, this follows from 5.8 (a) and the assumption that $x_{1,1}^{-} \overline{x_{2,0} .} m \in W$. For $H_{-1}$, it suffices similarly to prove that $x_{1,-1}^{-} x_{2,0}$. $m \in W$. By assumption, we can write

$$
x_{1,1}^{-} x_{2,0 .}^{-} m=A x_{1,0}^{-} x_{2,0}^{-} . m+B x_{2,0}^{-} x_{1,0}^{-} . m,
$$

for some $A, B \in \mathbb{C}$. If $A \neq 0$, applying $H_{-1}$ to both sides of (17) gives the desired conclusion. If $A=0$, we use the same argument with $H_{-2}$ to get $\overline{x_{1,-1}} \overline{x_{2,0}} \cdot m$ $\in W$.

To prove that (ii) implies (iii), note that, by $1.4, M_{\mu-\alpha_{1}-\alpha_{2}}$ is spanned by $\left\{x_{i, r}^{-} x_{j, s}^{-} . m\right\}_{i, j=1,2, r, s \in Z}$. Hence, $M_{\mu-\alpha_{1}-\alpha_{2}}=W$. Since $W \subseteq U_{q}(\mathfrak{g}) . m \cong V(\mu)$, $m_{\mu-\alpha_{1}-\alpha_{2}}(M)=0$.

For (iii) implies (i), suppose for a contradiction that $x_{1,1}^{-} x_{2,0}^{-} . m \notin W$. Then $\operatorname{dim}\left(M_{\mu-\alpha_{1}-\alpha_{2}}\right) \geq 3$. But, $\operatorname{dim}\left(V(\mu)_{\mu-\alpha_{1}-\alpha_{2}}\right)=2$. Since $m_{\mu-\alpha_{t}}(M)=0$ for $i$ $=1,2$, we must have $m_{\mu-\alpha_{1}-\alpha_{2}}(M)>0$.

\section{§6. $\mathbb{U}_{q}(\mathfrak{g})$-structure: a First Reduction}

The purpose of this section and the next is to prove

Theorem 6.1. Let $\lambda \in P^{+}$and let $V(\mathbf{P})$ be a minimal affinization of $\lambda$. Then, as a representation of $U_{q}(\mathfrak{g})$,

$$
V(\mathbb{P}) \cong \bigotimes_{r=0}^{\operatorname{int}\left(\frac{1}{2} \lambda(2)\right)} V\left(\lambda-2 r \lambda_{2}\right)
$$

Here, for any real number $b$, int $(b)$ is the greatest integer less than or equal to $b$.

The proof of Theorem 6.1 is by induction on $\lambda(2)$. The case $\lambda(2)=0$ was 
proved in 5.7. Note also that the minimal affinization of $\lambda_{2}$ is irreducible as a representation of $U_{q}(\mathrm{~g})$ since there is no $\mu \in P^{+}$such that $\mu<\lambda_{2}$.

For the inductive step, we shall need the following lemma. Recall that, if $V$ is any representation of $U_{q}(\hat{\mathrm{g}})$, its left dual ${ }^{t} V$ is the representation of $U_{q}(\hat{\mathrm{g}})$ on the vector space dual of $V$ given by

$$
\langle a . f, v\rangle=\langle f, S(a) . v\rangle, \quad\left(a \in U_{q}(\hat{\mathfrak{g}}), v \in V, f \in{ }^{t} V\right)
$$

where $S$ is the antipode of $U_{q}(\hat{\mathfrak{g}})$ and $\langle, \quad>$ is the natural pairing between $V$ and its dual. The right dual $V^{t}$ is defined in the same way, replacing $S$ by $S^{-1}$. Left and right duals of representations of $U_{q}(\mathrm{~g})$ are defined similarly. Clearly the (left or right) dual of an irreducible representation is again irreducible. In fact, it is well known that, for any $\lambda \in P^{+}$,

$$
{ }^{t} V(\lambda) \cong V(\lambda)^{t} \cong V\left(-w_{0} \lambda\right)
$$

where $w_{0}$ is the longest element of the Weyl group of $\mathfrak{g}$.

We introduce the following notation: for any $U_{q}(\mathfrak{g})$-module $W=\oplus W_{\lambda}$ of type 1 , set

$$
W_{\lambda}^{+}=\left\{w \in W_{\lambda} \mid x_{\imath}^{+} . w=0 \text { for all } i \in I\right\}
$$

Lemma 6.2. (i) For any $a \in \mathbf{C}^{\times}$,

$$
V\left(\lambda_{2}, a\right)^{t} \cong V\left(\lambda_{2}, a q^{6}\right), \quad{ }^{t} V\left(\lambda_{2}, a\right) \cong V\left(\lambda_{2}, a q^{-6}\right)
$$

(ii) For any $a, b \in \mathbb{C}^{\times}$,

$$
\operatorname{dim}\left(\left(V\left(\lambda_{2}, a\right) \otimes V\left(\lambda_{2}, b\right)\right)_{0}^{+}\right)=1
$$

Moreover, if. $0 \neq v_{0} \in\left(V\left(\lambda_{2}, a\right) \otimes V\left(\lambda_{2}, b\right)\right)_{0}^{+}$and $a / b \neq q^{ \pm 6}$, then $x_{0}^{ \pm} . v_{0}$ is a non-zero multiple of $v_{\mp \lambda_{2}} \otimes v_{\mp \lambda_{2}}$.

Proof. (i) Since, for any representation $V$ of $U_{q}(\hat{\mathfrak{g}})$, the canonical isomorphism of vector spaces ${ }^{t} V^{t} \rightarrow V$ is an isomorphism of representations, it suffices to prove the first formula. Since $V\left(\lambda_{2}\right)$ is a self-dual representation of $U_{q}(\mathfrak{g})$, we have a priori that $V\left(\lambda_{2}, a\right)^{t} \cong V\left(\lambda_{2}, b\right)$ for some $b \in \mathbf{C}^{\times}$.

Fix $v_{-\lambda_{2}}=x_{2}^{-} x_{1}^{-} x_{2}^{-} \cdot v_{\lambda_{2}}$. Then, $v_{-\lambda_{2}}$ is a non-zero element of $V\left(\lambda_{2}, a\right)_{-\lambda_{2}}$ and, for weight reasons,

$$
x_{0}^{+} . v_{\lambda_{2}}=A v_{-\lambda_{2}}
$$


for some $A \in \mathbf{C}$. Let $0 \neq v_{\lambda_{2}}^{t} \in V\left(\lambda_{2}, a\right)_{\lambda_{2}}^{t}$. Then $\left\langle v_{\lambda_{2}}^{t}, w\right\rangle=0$ if $w \notin V\left(\lambda_{2}, a_{2}\right)_{-\lambda_{2}}$. Normalize $v_{\lambda_{2}}^{t}$ so that

$$
\left\langle v_{\lambda_{2}}^{t}, v_{-\lambda_{2}}\right\rangle=1
$$

and let $v_{-\lambda_{2}}^{t}=x_{2}^{-} x_{1}^{-} x_{2}^{-} \cdot v_{\lambda_{2}}^{t}$. Again, for weight reasons, one has

$$
x_{0}^{+} . v_{\lambda_{2}}^{t}=B v_{-\lambda_{2}}^{t}
$$

for some $B \in \mathbb{C}$. Moreover, from the formula for $x_{0}^{+}$in 1.3 , it is clear that

$$
A=a^{-1} c, \quad B=b^{-1} c,
$$

where $c \in \mathbb{C}^{\times}$depends only on $q$, and not on $a$ or $b$. Thus, $A / B=b / a$. But $A / B$ may be computed as follows:

$$
\left\langle x_{0}^{+} . v_{\lambda_{2}}^{t}, v_{\lambda_{2}}\right\rangle=\left\langle v_{\lambda_{2}}^{t}, S^{-1}\left(x_{0}^{+}\right), v_{\lambda_{2}}\right\rangle=\left\langle v_{\lambda_{2}}^{t},-k_{0}^{-1} x_{0}^{+}, v_{\lambda_{2}}\right\rangle
$$

Hence,

$$
B\left\langle x_{2}^{-} x_{1}^{-} x_{2}^{-} . v_{\lambda_{2}}^{t}, v_{\lambda_{2}}\right\rangle=-q^{-2} A
$$

Since

$$
S\left(x_{2}^{-} x_{1}^{-} x_{2}^{-}\right) . v_{\lambda_{2}}=-q^{4} x_{2}^{-} x_{1}^{-} x_{2}^{-} \cdot v_{2}=-q^{4} v_{-\lambda_{2}},
$$

we find that $A / B=q^{6}$, and part (i) is proved.

(ii) Since $V\left(\lambda_{2}\right)$ is a self-dual representation of $U_{q}(\mathrm{~g})$, it follows that

$$
\operatorname{dim}\left(\left(V\left(\lambda_{2}, a\right) \otimes V\left(\lambda_{2}, b\right)\right)_{0}^{+}\right)=1
$$

If $x_{0}^{ \pm} \cdot v_{0}=0$, then C. $v_{0}$ is a $U_{q}(\hat{\mathfrak{g}})$-subrepresentation of the tensor product, and hence by (i) we have $a / b=q^{ \pm 6}$.

For the remainder of this paper, we assume that $\lambda \in P^{+}, \lambda(2) \geq 1$ and that 6.1 is known for $\lambda-\lambda_{2}$. We shall also assume that $\lambda(1) \geq 1$. The proof when $\lambda(1)=0$ is similar and easier.

We also fix for the rest of the paper an element $\mathbb{P}=\left(P_{i}\right)_{i \in I} \in \mathscr{P}^{\lambda}$ such that the roots of $P_{i}$ form a string with centre $a_{i}$ and length $\lambda(i), i=1,2$, and such that 


$$
\frac{a_{1}}{a_{2}}=q^{-(2 \lambda(1)+\lambda(2)+3)}
$$

Define an element $\mathbf{Q} \in \mathscr{P}^{\lambda-\lambda_{2}}$ by

$$
Q_{1}=P_{1}, Q_{2}=\prod_{i=1}^{\lambda(2)-1}\left(1-a_{2}^{-1} q^{-(\lambda(2)-2 \imath-1)} u\right)
$$

By 5.1, $V(\mathbf{P})$ and $V(\mathbf{Q})$ are minimal affinizations of $\lambda$ and $\lambda-\lambda_{2}$, respectively. In particular, 6.1 is known for $V(\mathbf{Q})$.

The main result proved in this section is:

Proposition 6.3. Let $\lambda, \mu \in P^{+}$and let $V(\mathbf{P})$ be a minimal affinization of $\lambda$ as above. Then: $\in \mathbf{N}$.

(i) $m_{\mu}(V(\mathbf{P})) \leq 1$ if $\mu$ is of the form $\lambda-r \theta-\alpha_{2}$ or $\lambda-r \theta-\alpha_{1}-\alpha_{2}$ for some $r$

(ii) $m_{\mu}(V(\mathbf{P})) \leq 2$ if $\mu$ is of the form $\lambda-r \theta$ for some $r \in \mathbf{N}$.

(iii) $m_{\mu}(V(\mathbf{P}))=0$ if $\mu$ is not of the form $\lambda-r \theta, \lambda-r \theta-\alpha_{2}$ or $\lambda-r \theta-\alpha_{1}-$ $\alpha_{2}$ for some $r \in \mathbf{N}$.

$$
\text { (iv) } m_{\lambda-r \theta}(V(\mathbf{P})) \geq 1 \text { for } 0 \leq r \leq \operatorname{int}\left(\frac{1}{2} \lambda(2)\right) \text {. }
$$

We deduce this from the next two results.

Lemma 6.4. For any $\lambda \in P^{+}$,

$V(\lambda) \otimes V\left(\lambda_{2}\right) \cong V\left(\lambda+\lambda_{2}\right) \oplus V\left(\lambda+\lambda_{2}-\alpha_{2}\right) \oplus V\left(\lambda+\lambda_{2}-\alpha_{1}-\alpha_{2}\right) \oplus V\left(\lambda+\lambda_{2}-\theta\right)$

Proof. By 2.1 (c), it suffices to prove the analogous classical result. We leave this to the reader.

Proposition 6.5. Let $\lambda \in P^{+}, \mathbf{P} \in \mathscr{P}^{\lambda}, \mathbf{Q} \in \mathscr{P}^{\lambda-\lambda_{2}}$ be as defined above.

(i) $V\left(\lambda_{2}, a_{2} q^{\lambda(2)-1}\right) \otimes V(\mathbf{Q})$ is generated as a representation of $U_{q}(\hat{\mathfrak{g}})$ by the tensor product of the highest weight vectors. In particular, $V(\mathbf{P})$ is isomorphic to a quotient of $V\left(\lambda_{2}, a_{2} q^{\lambda(2)-1}\right) \otimes V(\mathbf{Q})$.

(ii) Let $\mathbf{P}_{(1)}=\left(P_{1}, 1\right)$. Then, there exists a surjective homomorphism of representations of $U_{q}(\hat{\mathfrak{g}})$

$$
\pi: V\left(\lambda_{2}, a_{2} q^{\lambda(2)-1}\right) \otimes V\left(\lambda_{2}, a_{2} q^{\lambda(2)-3}\right) \otimes \cdots \otimes V\left(\lambda_{2}, a_{2} q^{-\lambda(2)+1}\right) \otimes V\left(\mathbf{P}_{(1)}\right) \rightarrow V(\mathbf{P})
$$

such that $\pi\left(v_{\lambda_{2}}^{\otimes \lambda(2)} \otimes v_{\mathbf{P}_{(1)}}\right)=v_{\mathbf{P}}$. 
We assume 6.5 for the moment and give the

Proof of 6.3. Parts (i), (ii) and (iii) are easy consequences of 6.4 and 6.5 (i) since 6.1 is known for $V(\mathbf{Q})$.

To prove (iv), we can assume that $\lambda(2) \geq 2$, since otherwise there is nothing to prove. Notice that, by 6.2 , we can (and do) choose elements $0 \neq w_{s} \in$ $\left(V\left(\lambda_{2}, a_{2} q^{\lambda(2)-4 s+3}\right) \otimes V\left(\lambda_{2}, a_{2} q^{\lambda(2)-4 s+1}\right)\right)_{0}^{+}$such that

$$
x_{0}^{-} \cdot w_{s}=v_{\lambda_{2}} \otimes v_{\lambda_{2}}
$$

For $1 \leq r \leq \operatorname{int}\left(\frac{1}{2} \lambda(2)\right)$, consider the element

$$
\begin{aligned}
& w=w_{1} \otimes w_{2} \otimes \cdots w_{r} \otimes v_{\lambda_{2}}^{\otimes \lambda(2)-2 r} \otimes v_{\mathbf{P}_{(1)}} \\
& \in V\left(\lambda_{2}, a_{2} q^{\lambda(2)-1}\right) \otimes V\left(\lambda_{2}, a_{2} q^{\lambda(2)-3}\right) \otimes \cdots \otimes V\left(\lambda_{2}, a_{2} q^{-\lambda(2)+1}\right) \otimes V\left(\mathbf{P}_{(1)}\right) .
\end{aligned}
$$

Clearly, $x_{i, 0 .}^{+} w=0$ for $i=1,2$, and an easy computation shows that

$$
\left(x_{0}^{-}\right)^{r} \cdot w=q^{r(r-1)}[r]_{q^{2}} v_{\lambda_{2}}^{\otimes \lambda(2)} \otimes v_{\mathbf{P}_{(1)}} .
$$

Hence, $\pi\left(\left(x_{0}^{-}\right)^{r} . w\right) \neq 0$ and so $\pi(w)$ is a non-zero element of $V(\mathbb{P})_{\lambda-r \theta}^{+}$. This proves 6.3 (iv).

Proof of 6.5. Assuming 6.5(i) we give the proof of 6.5 (ii). The proof is by induction on $\lambda(2)$. The case $\lambda(2)=1$ is just 6.5 (i). So if $\lambda(2)>1$, by the induction hypothesis applied to $\lambda-\lambda_{2}$, we have a surjective homomorphism of representations of $U_{q}(\hat{\mathfrak{g}})$

$$
\pi^{\prime}: V\left(\lambda_{2}, a_{2} q^{\lambda(2)-3}\right) \otimes \cdots \otimes V\left(\lambda_{2}, a_{2} q^{-\lambda(2)+1}\right) \otimes V\left(\mathbf{P}_{(1)}\right) \rightarrow V(\mathbf{Q})
$$

\section{Consider}

$$
\mathrm{id} \otimes \pi^{\prime}: V\left(\lambda_{2}, a_{2} q^{\lambda(2)-1}\right) \otimes \cdots \otimes V\left(\lambda_{2}, a_{2} q^{-\lambda(2)+1}\right) \otimes V\left(\mathbf{P}_{(1)}\right) \rightarrow V\left(\lambda_{2}, a_{2} q^{\lambda(2)-1}\right) \otimes V(\mathbf{Q}) .
$$

By $6.5(\mathrm{i})$, the right-hand side has $V(\mathbb{P})$ as a quotient and so we get the required surjective homomorphism

$$
\pi: V\left(\lambda_{2}, a_{2} q^{\lambda(2)-1}\right) \otimes \cdots \otimes V\left(\lambda_{2}, a_{2} q^{-\lambda(2)+1}\right) \otimes V\left(\mathbf{P}_{(1)}\right) \rightarrow V(\mathbb{P})
$$

We now prove $6.5(\mathrm{i})$. Let $M=U_{q}(\hat{\mathrm{g}}) .\left(v_{\lambda_{2}} \otimes v_{\mathbf{Q}}\right)$. We first show that it suffices to prove 


$$
m_{\mu}(M)=m_{\mu}\left(V\left(\lambda_{2}, a_{2} q^{\lambda(2)-1}\right) \otimes V(\mathbf{Q})\right) \quad \text { for } \quad \mu>\lambda-\theta
$$

To see this, assume that $M$ is a proper subrepresentation of the tensor product and let $N$ be the corresponding quotient. It follows from 6.4 and (18) that

$$
m_{\mu}(N)=0 \text { unless } \mu \leq \lambda-\theta
$$

On the other hand, dualizing the projection map

$$
V\left(\lambda_{2}, a_{2} q^{\lambda(2)-1}\right) \otimes V(\mathbf{Q}) \rightarrow N
$$

we get a non-zero (hence injective) homomorphism of representations of $U_{q}(\hat{\mathfrak{g}})$

$$
V(\mathbf{Q}) \rightarrow V\left(\lambda_{2}, a_{2} q^{\lambda(2)-1}\right)^{t} \otimes N
$$

It follows that

$$
m_{\lambda-\lambda_{2}}\left(V\left(\lambda_{2}\right) \otimes N\right) \geq 1
$$

and hence by 6.4 that $m_{\lambda-\theta}(N)>0$.

Note that the preceding argument proves that, if $M^{\prime}$ is any $U_{q}(\hat{\mathfrak{g}})$-subrepresentation of $V\left(\lambda_{2}, a_{2} q^{\lambda(2)-1}\right) \otimes V(\mathbf{Q})$ containing $M$, and if $N^{\prime}$ is the corresponding quotient of the tensor product, then $m_{\lambda-\theta}\left(N^{\prime}\right)>0$. In particular, any irreducible quotient $N^{\prime}$ of $N$ must have $m_{\lambda-\theta}\left(N^{\prime}\right)>0$. Taking $N^{\prime}$ to be an affinization $V(\mathbf{R})$, say, of $\lambda-\theta$, we have a surjective map of $U_{q}(\hat{\mathfrak{g}})$-representations

$$
V\left(\lambda_{2}, a_{2} q^{\lambda(2)-1}\right) \otimes V(\mathbf{Q}) \rightarrow V(\mathbf{R})
$$

and hence, dualizing, an injective map

$$
V(\mathbf{Q}) \rightarrow V\left(\lambda_{2}, a_{2} q^{\lambda(2)-1}\right)^{t} \otimes V(\mathbf{R})=V\left(\lambda_{2}, a_{2} q^{\lambda(2)+5}\right) \otimes V(\mathbf{R})
$$

by 6.2. The highest weight vector in $V(\mathbf{Q})$ must map to (a non-zero multiple of) the tensor product of the highest weight vectors on the right-hand side. But this is impossible, since $a_{2} q^{\lambda(2)+5}$ is not a root of $Q_{2}$. Hence, $N=0$ and part (i) is proved.

We now prove (18). The statement is obviously true for $\mu=\lambda$. For $\mu=\lambda$ $-\alpha_{1}$, the statement follows from 6.4 and the fact that 6.1 is known for $V(\mathbf{Q})$. For $\mu=\lambda-\alpha_{2}$ or $\lambda-2 \alpha_{2}$, notice that, by 5.2 (b) it suffices to prove the result for $U_{q}\left(\widehat{s}_{2}\right)$. But this follows from 4.9 (a) of [3] since 


$$
V\left(\lambda_{2}, a_{2} q^{\lambda(2)-1}\right)_{(2)} \otimes V(\mathrm{Q})_{(2)} \cong V\left(1, a_{2} q^{\lambda(2)-1}\right) \otimes V\left(\lambda(2)-1, a_{2} q^{-1}\right)
$$

as representations of $\widehat{U}_{2}$.

Finally, we must prove (18) for $\lambda-\alpha_{1}-\alpha_{2}$. For this, it obviously suffices to prove that

$$
\left(V\left(\lambda_{2}, a_{2} q^{\lambda(2)-1}\right) \otimes V(\mathbf{Q})\right)_{\lambda-\alpha_{1}-\alpha_{2}} \subseteq M
$$

The left-hand side is spanned by

$$
\left\{x_{1}^{-} x_{2}^{-} \cdot v_{\lambda_{2}} \otimes v_{\mathbf{Q}}, v_{\lambda_{2}} \otimes x_{1}^{-} x_{2}^{-} \cdot v_{\mathbf{Q}}, v_{\lambda_{2}} \otimes x_{2}^{-} x_{1}^{-}, v_{\mathbf{Q}}, x_{2}^{-}, v_{\lambda_{2}} \otimes x_{1}^{-}, v_{\mathbf{Q}}\right\}
$$

Now, since $m_{\lambda}(M)$ and $m_{\lambda-\alpha_{2}}(M)$ are both strictly positive, $M$ contains $x_{2}^{-} \cdot v_{\lambda_{2}} \otimes$ $v_{\mathbf{Q}}$ and $v_{\lambda_{2}} \otimes x_{2}^{-} \cdot v_{\mathbf{Q}}$ ( since $M$ contains two linear combinations of these vectors which are nor scalar multiples of each other). Also, $M$ contains

$$
v_{\lambda_{2}} \otimes x_{1}^{-} \cdot v_{\mathbf{Q}}=x_{1}^{-} .\left(v_{\lambda_{2}} \otimes v_{\mathbf{Q}}\right)
$$

It follows that $M$ contains the three vectors

$$
x_{1}^{-} \cdot\left(x_{2}^{-} \cdot v_{\lambda_{2}} \otimes v_{\mathbf{Q}}\right), \quad x_{1}^{-} \cdot\left(v_{\lambda_{2}} \otimes x_{2}^{-} \cdot v_{\mathbf{Q}}\right), \quad x_{2}^{-} \cdot\left(v_{\lambda_{2}} \otimes x_{1}^{-} \cdot v_{\mathbf{Q}}\right),
$$

i.e. that $M$ contains the three vectors

$$
\begin{gathered}
v_{\lambda_{2}} \otimes x_{1}^{-} x_{2}^{-} \cdot v_{\mathbf{Q}}, \\
x_{1}^{-} x_{2}^{-} \cdot v_{\lambda_{2}} \otimes v_{\mathbf{Q}}+q^{-2} x_{2}^{-} \cdot v_{\lambda_{2}} \otimes x_{1}^{-} \cdot v_{\mathbf{Q}}, \\
x_{2}^{-} \cdot v_{\lambda_{2}} \otimes x_{1}^{-} \cdot v_{\mathbf{Q}}+q^{-1} v_{\lambda_{2}} \otimes x_{2}^{-} x_{1}^{-} \cdot v_{\mathbf{Q}} .
\end{gathered}
$$

Since these vectors are obviously linearly independent, it suffices to prove that

$$
\left[x_{2}^{+}, x_{0}^{+}\right] .\left(v_{\lambda_{2}} \otimes v_{\mathbf{Q}}\right)
$$

is linearly independent of the vectors in (19).

To compute the vector in (20), we need the following formulas:

$$
\begin{gathered}
x_{1,1}^{-} \cdot v_{\mathbf{Q}}=a_{1}^{-1} q^{2 \lambda(1)-2} x_{1}^{-} \cdot v_{\mathbf{Q}}, \\
x_{1,1}^{-} x_{2}^{-} \cdot v_{\mathbf{Q}}=a_{1}^{-1} q^{2 \lambda(1)-2} x_{1}^{-} x_{2}^{-} v_{\mathbf{Q}} .
\end{gathered}
$$

By the formula for the isomorphism $f$ in 1.3 , 


$$
\left[x_{2}^{+}, x_{0}^{+}\right]=c\left[x_{2}^{-}, x_{1,1}^{-}\right]_{q}\left(k_{1} k_{2}\right)^{-1},
$$

where $c \in \mathbf{C}^{\times}$, and for any $x, y \in U_{q}(\hat{\mathrm{g}})$, we define

$$
[x, y]_{q}=q x y-q^{-1} y x
$$

Using this, we find that

$$
\begin{aligned}
{\left[x_{2}^{+}, x_{0}^{+}\right] .\left(v_{\lambda_{2}} \otimes v_{\mathbf{Q}}\right)=} & c q^{-(2 \lambda(1)+\lambda(2))}\left(q^{-1}\left[x_{2}^{-}, x_{1,1}^{-}\right]_{q} \cdot v_{\lambda_{2}} \otimes v_{\mathbf{Q}}+v_{\lambda_{2}} \otimes\left[x_{2}^{-}, x_{1,1}^{-}\right]_{q} \cdot v_{\mathbf{Q}}\right) \\
= & c q^{-(2 \lambda(1)+\lambda(2))}\left(-a_{2}^{-1} q^{-\lambda(2)+1} x_{1}^{-} x_{2}^{-} \cdot v_{\lambda_{2}} \otimes v_{\mathbf{Q}}\right. \\
& \left.\quad+v_{\lambda_{2}} \otimes\left(a_{1}^{-1} q^{2 \lambda(1)-1} x_{2}^{-} x_{1}^{-} \cdot v_{\mathbf{Q}}-a_{2}^{-1} q^{\lambda(2)-3} x_{1}^{-} x_{2}^{-} \cdot v_{\mathbf{Q}}\right)\right) .
\end{aligned}
$$

An easy computation shows that this is linearly dependent on the vectors in (20) iff

$$
\frac{a_{1}}{a_{2}}=q^{2 \lambda(1)+\lambda(2)+2},
$$

contradicting our assumption that $a_{1} / a_{2}=q^{-(2 \lambda(1)+\lambda(2)+2)}$.

The proof of (21) is easy since we know from 5.1 (a) and 5.4 (b) that $x_{1,1}^{-}, v_{\mathbf{Q}}$ must be a scalar multiple of $x_{1}^{-} . v_{\mathbf{Q}}$. As for (22), observe that by 5.1 (a) and 5.4 (b) again we know a priori that

$$
x_{1,1}^{-} x_{2}^{-} \cdot v_{\mathbf{Q}}=A x_{1}^{-} x_{2}^{-} \cdot v_{\mathbf{Q}}+B x_{2}^{-} x_{1}^{-} \cdot v_{\mathbf{Q}}
$$

for some $A, B \in \mathbf{C}$. Applying $x_{1}^{+}$and $x_{2}^{+}$to both sides of gives the pair of equations

$$
\begin{aligned}
A[\lambda(2)-1]_{q}+B[\lambda(2)]_{q} & =[\lambda(2)-1]_{q} a_{1}^{-1} q^{2 \lambda(1)-2}, \\
A[\lambda(1)+1]_{q^{2}}+B[\lambda(1)]_{q^{2}} & =q^{2 \lambda(1) a_{1}^{-1}}[\lambda(1)]_{q^{2}}+a_{2}^{-1} q^{(2 \lambda(1)+\lambda(2)+1)} .
\end{aligned}
$$

Using $a_{1} / a_{2}=q^{-(2 \lambda(1)+\lambda(2)+3)}$, we find that the unique solution is $A=a_{1}^{-1} q^{2 \lambda(1)-2}, B$ $=0$.

\section{§7. Completion of the Proof of Theorem 6.1}

In view of 6.5 , to complete the proof of 6.1 , it suffices to establish

Proposition 7.1. Let $\lambda \in P^{+}$and let $V(\mathbf{P})$ be a minimal affinization of $\lambda$. 
Then:

(i ) $m_{\lambda-r \theta}(V(\mathbf{P}))=1$ if $0 \leq r \leq$ int $\left(\frac{1}{2} \lambda(2)\right)$.

(ii) $m_{\mu}(V(\mathbb{P}))=0$ if $\mu$ is of the form $\lambda-r \theta-\alpha_{2}$, for some $r \in \mathbb{N}$.

(iii) $m_{\mu}(V(\mathbb{P}))=0$ if $\mu$ is of the form $\lambda-r \theta-\alpha_{1}-\alpha_{2}$, for some $r \in \mathbb{N}$.

We need three lemmas.

Lemma 7.2. Suppose that there exists $0 \neq v \in V(\mathbb{P})_{\mu}^{+}$such that

$$
x_{1,1}^{+} . v=x_{1,-1}^{+} . v=0
$$

(resp. $\left.x_{2,1}^{+} . v=x_{2,-1}^{+} . v=0\right)$. Assume that $m_{\mu+\alpha_{i}}(V(\mathbf{P}))=0$ for $i=1,2$. Then, $\lambda$ $=\mu$.

Proof. We prove, by induction on $k \in \mathbf{N}$, that

$$
x_{i, \pm k}^{+} v=0 \text { for all } i=1,2 \text {. }
$$

It is easy to see using the relations in 1.3 that the $k_{j}$ and $h_{j, s}$ preserve the finite-dimensional space

$$
V(\mathbb{P})_{\mu}^{++}=\left\{w \in V(\mathbb{P})_{\mu}: x_{i, k}^{+} . w=0 \text { for all } i \in I, k \in \mathbb{Z}\right\}
$$

It follows that there exists a $U_{q}(\hat{\mathfrak{g}})$-highest weight vector in $V(\mathbb{P})_{\mu}$, which is possible only if $\lambda=\mu$.

It is obvious that (23) holds when $k=0$. We assume that it holds for $k$ and prove it for $k+1$. Using the relations in 1.3 , we find that

$$
x_{j, 0}^{+} x_{i, \pm(k+1)}^{+} \in U_{q}(\hat{\mathrm{g}}) x_{j, 0}^{+}+U_{q}(\hat{\mathrm{g}}) x_{j, \pm 1}^{+}+U_{q}(\hat{\mathrm{g}}) x_{i, \pm k}^{+},
$$

and hence by the induction hypotheses we see that $x_{i, \pm(k+1)}^{+} . v \in V(\mathbb{P})_{\mu+\alpha_{k}}^{+}$. Since $m_{\mu+\alpha_{\mathrm{t}}}(V(\mathbb{P}))=0$ by assumption, this forces $x_{i, \pm(k+1)}^{+} \cdot v=0$, establishing (23) for $k+1$.

Lemma 7.3. Let $0 \neq v \in V(\mathbb{P})_{\mu}^{+}$be such that $x_{1, s^{\prime} . v}^{+}=0$ if $0 \leq s^{\prime}<s$ or if $s$ $<s^{\prime} \leq 0$. Then

(i ) $\left(x_{2,0}^{+}\right)^{3} x_{1, s}^{+} \cdot v=0$,

(ii) $x_{1,0}^{+} x_{2,0}^{+} x_{1, s .}^{+} v=0$, 
(iii) $x_{1,0}^{+}\left(x_{2,0}^{+}\right)^{2} x_{1, s .}^{+} v \in V(\mathbf{P})_{\mu+2 \alpha_{1}+2 \alpha_{2}}^{+}$.

Proof. Using the quantized Serre relations in 1.3, we find that

$$
\left(x_{2,0}^{+}\right)^{3} x_{1, \pm s}^{+} \in U_{q}(\hat{\mathfrak{g}}) . x_{2,0}^{+} .
$$

Part (i) is now immediate.

For (ii), it suffices to notice that the relations in 1.3 give

$$
x_{1,0}^{+} x_{2,0}^{+} x_{1, \pm s}^{+} \in U_{q}(\hat{\mathfrak{g}}) x_{2,0}^{+}+\sum_{0 \leq s^{\prime}<s} U_{q}(\hat{\mathfrak{g}}) x_{1, \pm s^{\prime}}^{+}
$$

if $s>0$.

For (iii), we use the following consequences of 1.3 :

$$
\begin{gathered}
\left(x_{1,0}^{+}\right)^{2} x_{2,0}^{+} \in U_{q}(\hat{\mathfrak{g}}) x_{1,0}^{+}, \\
x_{2,0}^{+} x_{1,0}^{+}\left(x_{2,0}^{+}\right)^{2} \in U_{q}(\hat{\mathrm{g}})\left(x_{2,0}^{+}\right)^{3}+U_{q}(\hat{\mathrm{g}}) x_{1,0}^{+}+U_{q}(\hat{\mathrm{g}}) x_{1,0}^{+} x_{2,0}^{+} .
\end{gathered}
$$

The result now follows from parts (i) and (ii).

Lemma 7.4. Let $\mu \in P^{+}$be such that

$$
m_{\mu+\eta}(V(\mathbb{P}))=0 \text { if } \eta \neq s \theta, s \in \mathbb{Z}_{+} .
$$

Then, $\left(x_{2,0}^{+}\right)^{2} x_{1, \pm 1}^{+}$maps $V(\mathbb{P})_{\mu}^{+}$to $V(\mathbb{P})_{\mu+\theta}^{+}$. Further, if $v \in V(\mathbb{P})_{\mu}^{+}$is such that $x_{1, \pm 1}^{+}, v \neq 0$, then

$$
\left(x_{2,0}^{+}\right)^{2} x_{1, \pm 1}^{+}, v \neq 0
$$

Proof. It is clear for weight reasons that $\left(x_{2,0}^{+}\right)^{2} x_{1, \pm 1}^{+}$maps $V(\mathbb{P})_{\mu}^{+}$to $V(\mathbb{P})_{\mu+\theta}$. Thus, it suffices to prove that

$$
x_{i, 0}^{+}\left(x_{2,0}^{+}\right)^{2} x_{1, \pm 1 .}^{+} v=0 \text { if } v \in V(\mathbb{P})_{\mu}^{+} .
$$

For $i=2$, this is just $7.3(\mathrm{i})$. For $i=1$, the result is obvious from 7.3 (iii) and (28).

Now suppose that $\left(x_{2,0}^{+}\right)^{2} x_{1,1}^{+} \cdot v=0 . \quad$ By $7.3(\mathrm{ii}), x_{1,0}^{+} x_{2,0}^{+} x_{1,1}^{+}, v=0$ as well and (28) now forces

$$
x_{2,0}^{+} x_{1,1 .}^{+} v=0 .
$$


Now the quantized Serre relations in 1.3 give $x_{1,0}^{+} x_{1,1}^{+}, v=0$ and so by a final application (28), we get

$$
x_{1,1}^{+} v=0
$$

One proves similarly that $\left(x_{2,0}^{+}\right)^{2} x_{1,-1}^{+} . v=0$ implies that $x_{1,-1}^{+} . v=0$, and the proof of 7.4 is now complete.

We are now in a position to give the

Proof of 7.1. All three parts are proved by induction on $r$. If $r=0$, the result follows from 5.1 (a). We assume that (i), (ii) and (iii) hold for $r$ and prove them for $r+1$.

(i) Suppose that $m_{\lambda-(r+1) \theta}(V(\mathbf{P}))>1$. Then, by 7.4 , there exists $0 \neq v_{0} \in$ $V(\mathbf{P})_{\lambda-(r+1) \theta}^{+}$such that $x_{1,1}^{+}, v=0$.

Suppose now that $x_{1,-1}^{+} . v \neq 0$. For $s=0,1, \ldots, r+1$, define $v_{s} \in V(\mathbb{P})$ by

$$
v_{s}=\left(\left(x_{2,0}^{+}\right)^{2} x_{1,-1}^{+}\right)^{s} \cdot v
$$

We claim that the $v_{s}$ have the following properties:

(i) $s \quad 0 \neq v_{s} \in V(\mathbf{P})_{\lambda-(r+1-s) \theta}^{+}$for all $0 \leq s \leq r+1$;

(ii) $s x_{i, k}^{+}, v_{s}=0$ for $i=1,2, k \geq 0$.

Note that $(\mathrm{i})_{0}$ holds by assumption and (ii) ${ }_{0}$ by the choice of $v_{0}$. Assuming that these properties hold for $s$ we now prove that they hold for $s+1$. Lemma 7.2 implies that $x_{1,-1}^{+}, v_{s} \neq 0$ if $0 \leq_{s} \leq_{r}$ and 7.4 now shows that $v_{s+1} \neq 0$. To prove that (ii) $s_{s+1}$ holds, observe that, by the proof of 7.2 , it suffices to prove that $x_{1,1}^{+}, v=0$. Using the relations in 1.3 we find that

$$
x_{1,1}^{+}\left(x_{2,0}^{+}\right)^{2} x_{1,-1}^{+} \in U_{q}(\hat{\mathfrak{g}}) x_{1,1}^{+} x_{2,0}^{+} x_{1,-1}^{+}+U_{q}(\hat{\mathfrak{g}}) x_{2,1}^{+} x_{2,0}^{+} x_{1,-1}^{+}+U_{q}(\hat{\mathfrak{g}}) x_{1,0}^{+} x_{2,0}^{+} x_{1,-1}^{+}
$$

The third term kills $v_{s}$ by 7.3 (ii); on the other hand, using 1.3 again, we find that the first two terms are contained in

$$
\sum_{i=1}^{2}\left(U_{q}(\hat{\mathfrak{g}}) x_{i, 0}^{+}+U_{q}(\hat{\mathfrak{g}}) x_{i, 1}^{+}\right)
$$

and hence kill $v_{s}$ as well. This proves the claim.

Note that $v_{r+1}=A v_{\mathbf{P}}$, for some $A \in \mathbf{C}^{\times}$. Since $\operatorname{dim}\left(V(\mathbb{P})_{\lambda-\alpha_{2}}\right)=1$, it follows 
that

$$
x_{2,0}^{+} x_{1,-1}^{+} \cdot v_{r}=B x_{2,0}^{-} \cdot v_{\mathbf{P}},
$$

for some $B \in \mathbf{C}^{\times}$. Applying $x_{2,1}^{+}$to both sides of this equation, and using 1.3 we find

$$
\begin{aligned}
x_{2,1}^{+} x_{2,0}^{+} x_{1,-1}^{+} . v_{r} & =q^{2} x_{2,0}^{+} x_{2,1}^{+} x_{1,-1 .}^{+} v_{r} \\
& \in q^{2} x_{2,0}^{+}\left(U_{q}(\hat{\mathrm{g}}) x_{2,1}^{+}+U_{q}(\hat{\mathrm{g}}) x_{2,0}^{+}+U_{q}(\hat{\mathrm{g}})\right) x_{1,0}^{+} \cdot v_{r} .
\end{aligned}
$$

Now, using (ii) $r$, we get

$$
0=B \phi_{2,1}^{+} v_{\mathbf{P}}
$$

But this is impossible, because from 2.3 we find that

$$
\phi_{2,1}^{+} v_{\mathbf{P}}=a_{2}^{-1} q_{2}^{-1}\left(q_{2}^{2 \lambda(2)}-1\right) v_{\mathbf{P}}
$$

which is non-zero since $\lambda(2)>0$. This completes the proof of 7.1 (i).

(ii) Suppose that $m_{\lambda-(r+1) \theta-\alpha_{2}}(V(\mathbf{P}))>0$. The induction hypothesis on $r$ implies that

$$
V(\mathbf{P})_{\lambda-r \theta-\eta}^{+}=0 \text { if } \eta=\alpha_{2}, 2 \alpha_{2}, 3 \alpha_{2} \text {, or } \alpha_{2}-\alpha_{1} \text {. }
$$

Let $0 \neq v \in V(\mathbf{P})_{\lambda-(r+1) \theta-\alpha_{2}}^{+}$. We shall prove that $v$ is actually $U_{q}(\hat{\mathfrak{g}})$-highest weight, which is obviously impossible. We first prove, by induction on $k$, that $x_{1, k}^{+}, v=0$. By (29), it suffices to prove that $x_{1, k+1}^{+} . v \in V(\mathbf{P})_{\lambda-r \theta-3 \alpha_{2}}^{+}$. Since $x_{1,0}^{+} x_{1, k+1}^{+} \in \sum_{0 \leq s<k+1} U_{q}(\hat{\mathfrak{g}}) x_{1, s}^{+}$, we see that

$$
x_{1,0 .}^{+} x_{1, k+1}^{+}, v=0
$$

To prove that $x_{2,0}^{+} x_{1, k+1}^{+} . v=0$, define $v^{\prime}=\left(x_{2,0}^{+}\right)^{2} x_{1, k+1}^{+} . v$ and $v^{\prime \prime}=x_{1,0}^{+} \cdot v^{\prime}$. Then, by (29),

$$
\begin{aligned}
& 7.3(\mathrm{iii}) \Longrightarrow v^{\prime \prime} \in V(\mathbf{P})_{\lambda-r \theta-\alpha_{2}+\alpha_{1}}^{+} \Longrightarrow v^{\prime \prime}=0, \\
& 7.3(\mathrm{i}) \Longrightarrow v^{\prime} \in V(\mathbf{P})_{\lambda-r \theta-\alpha_{2}} \Longrightarrow v^{\prime}=0, \\
& 7.3(\mathrm{ii}) \Longrightarrow x_{2,0}^{+} x_{1, k+1}^{+} \cdot v \in V(\mathbf{P})_{\lambda-r \theta-3 \alpha 2}^{+} \Longrightarrow x_{2,0}^{+} x_{1, k+1}^{+} . v=0 .
\end{aligned}
$$

To prove that $x_{2, k}^{+} . v=0$, we again proceed by induction on $k$. We assume 
that $k \geq 0$; the proof for $k \leq 0$ is similar.

Using 1.3 and the fact that $x_{1, k}^{+}$. $v=0$ for all $k$, we see that

$$
\begin{aligned}
& x_{1, r}^{+} x_{2, k+1}^{+} v=0, \text { for } r=-1,0, \text { and } 1, \\
& x_{2,0}^{+} x_{2, k+1}^{+} v=0 .
\end{aligned}
$$

But now 7.2 implies that $x_{2, k+1}^{+} v=0$ (since $\left.\lambda-(r+1) \theta \neq \lambda\right)$. This completes the proof of 7.1 (ii).

(iii) Let $v \in V(\mathbb{P})_{\lambda-(r+1) \theta-\alpha_{1}-\alpha_{2}}^{+}$. Since

$$
m_{\lambda-(r+1) \theta-\alpha_{i}}(V(\mathbb{P}))=0 \text {, for } i=1,2 \text {, }
$$

and since $\lambda \neq \lambda-(r+1) \theta-\alpha_{1}-\alpha_{2}$, it suffices by 7.2 to prove that $x_{2, \pm 1}^{+}, v=0$. To do this, note that by 6.3 , it is enough to prove that $x_{2, \pm 1}^{+} . v \in V(\mathbb{P})_{\lambda-(r+1) \theta-\alpha_{1}}^{+}$. Clearly, by $1.3, x_{2,0}^{+} x_{2, \pm 1}^{+} . v=0$.

To prove that $x_{1,0}^{+} x_{2, \pm 1}^{+} . v=0$, it suffices by 7.2 to prove that

$$
\begin{gathered}
x_{1,0}^{+} \cdot x_{2, \pm 1 \cdot}^{+} \cdot v \in V(\mathbb{P})_{\lambda-(r+1) \theta}^{+} \\
x_{1, s .}^{+} \cdot x_{1,0}^{+} x_{2, \pm 1}^{+} \cdot v=0 \text { for } s= \pm 1 .
\end{gathered}
$$

The fact that $\left(x_{1,0}^{+}\right)^{2} x_{2, \pm 1}^{+} . v=0$ is clear from the quantized Serre relations in 1.3. By using this and the other relations in 1.3 it is easy to see that $x_{2,0}^{+} x_{1,0}^{+} x_{2, \pm 1}^{+} v$ $\in V(\mathbb{P})_{\lambda-r \theta-\alpha_{1}-\alpha_{2}}$, and hence must be zero by 6.3. This proves (30).

To prove (31), one checks first, using 1.3 , that

$$
\left(x_{2,0}^{+}\right)^{2} x_{1, s}^{+} x_{1,0}^{+} x_{2, \pm 1}^{+} \in U_{q}(\hat{\mathfrak{g}}) \cdot x_{2,0}^{+} x_{1,0}^{+} x_{2, \pm 1}^{+}+U_{q}(\hat{\mathfrak{g}}) \cdot x_{2,0}^{+} x_{2, \pm 1}^{+} .
$$

It follows that $\left(x_{2,0}^{+}\right)^{2} x_{1, s}^{+} x_{1,0}^{+} x_{2, \pm 1}^{+}, v=0$ for $s=0,1$. Lemma 7.4 now implies that in fact

$$
x_{1, s}^{+} x_{1,0}^{+\prime} x_{2, \pm 1 .}^{+^{\prime}} v=0 \text { for } s= \pm 1 .
$$

This completes the proof of 7.1 (iii).

The proof of Theorem 6.1 is now complete.

\section{References}

[1] Beck, J., Braid group action and quantum affine algebras, preprint, MIT, 1993. 
[2] Chari, V. and Pressley, A. N., New unitary representations of loop groups, Math. Ann., 275 (1986), 87-104.

[3] - Quantum affine algebras, Commun. Math. Phys., 142 (1991), 261-83.

[4] - Small representations of quantum affine algebras, Lett. Math. Phys., 30 (1994). 131-45.

[5] - A Guide to Quantum Groups, Cambridge University Press, Cambridge, 1994.

[6] - Quantum affine algebras and their representations, preprint, 1994.

[7] Delius, G. W. and Zhang, Y.-Z., Finite-dimensional representations of quantum affine algebras, preprint, 1994.

[8] Drinfel'd, V. G., A new realization of Yangians and quantized affine algebras, Soviet Math. Dokl., 36 (1988), 212-6.

[9] Frenkel, I. B. and Reshetikhin, N. Yu., Quantum affine algebras and holonomic difference equations, Commun. Math. Phys., 146 (1992), 1-60.

[10] Lusztig, G., Introduction to Quantum Groups, Progress in Mathematics 110. Birkhäuser. Boston, 1993. 
